\title{
Pseudochirality at exceptional rings of optical metasurfaces
}

\author{
Radoslaw Kolkowski $\odot$, Stefanos Kovaios, and A. Femius Koenderink $๑^{*}$ \\ Center for Nanophotonics, AMOLF, Science Park 104, 1098XG Amsterdam, The Netherlands
}

(Received 21 September 2020; accepted 18 May 2021; published 4 June 2021)

\begin{abstract}
Non-Hermitian optical metasurfaces have recently attracted interest as a platform for controlling light in amplitude, phase, and polarization. Here we predict that breaking parity-time symmetry in honeycomblike active plasmonic metasurfaces can lead to nonunitary circular dichroism at oblique incidence. This extraordinary chiroptical response is achieved through band folding that enables coupling of incident light to spin-polarized flat bands surrounded by exceptional rings, formed at Dirac points of plasmonic lattice resonances. The reported spin polarization differs from previously reported chiral and pseudochiral phenomena in that it solely emerges from spatial distribution of gain and loss and at the same time it does not involve the inherent chirality of isolated exceptional points, but instead it can be considered as a non-Hermitian counterpart of spin polarization of $K$-point valleys. Our findings may become useful in designing and realizing novel polarization-controlling optical elements and spin-polarized exceptional ring lasers.
\end{abstract}

DOI: 10.1103/PhysRevResearch.3.023185

\section{INTRODUCTION}

Metasurfaces are two-dimensional arrays of resonant scatterers offering diverse light-shaping functions [1-4]. Recently, non-Hermitian metasurfaces with spatially patterned gain and/or loss have attracted significant interest, promising unprecedented control of optical fields [5-13]. These capabilities are mainly associated with exceptional points (EPs), where two distinct complex eigenstates merge into one due to breaking parity-time $(\mathcal{P} \mathcal{T})$ symmetry [14-17]. The most peculiar property of non-Hermitian systems is their directional response, which has been demonstrated as unidirectional reflectivity [18,19], nonreciprocal transport [20], on-chip controllable directional lasing in photonic crystal cavities [21] and microring resonators [22], vortex beam generation [23], chiral response [24], asymmetric mode switching [25], and polarization state conversion [26]. Among the above examples, controlling the polarization of light is probably the most natural function offered by non-Hermitian metasurfaces [27-32] due to inherent chirality of photonic eigenstates in the vicinity of EPs [33-40].

An emblematic model system for $\mathcal{P} \mathcal{T}$-symmetry breaking is the honeycomb lattice with gain and loss treated in the tightbinding Hamiltonian model (i.e., lattice model with coupling limited to nearest neighbors). Within this model lattices are predicted to support exceptional rings (ERs): closed contours of EPs in momentum space, created upon non-Hermitian perturbation at Dirac points [41-43]. The tight-binding model

\footnotetext{
*f.koenderink@amolf.nl

Published by the American Physical Society under the terms of the Creative Commons Attribution 4.0 International license. Further distribution of this work must maintain attribution to the author(s) and the published article's title, journal citation, and DOI.
}

and concomitant ERs have been recreated in waveguide arrays $[44,45]$, to which tight-binding Hamiltonians are directly applicable through the paraxial approximation (i.e., simplifying the Helmholtz equation to a Schrödinger-like equation), wherein the waveguide axis plays the role of time. The closest nonparaxial realization would be a honeycomb lattice of resonant scatterers with modulated loss and gain. In spite of extensive work on such nonparaxial honeycomb lattices, including photonic crystals and metasurfaces [46-54], the farfield scattering response of $K$-point ERs in such systems has not yet been investigated. An important factor may be that tight-binding models, commonly employed for dense plasmon particle lattices, intrinsically cannot account for far fields as such a description is electrostatic and intrinsically lacks far-field input and output channels. However, the far-field signatures of $K$-point ERs are of high significance, first, as a possible telltale of non-Hermitian band topology [39] through polarization properties of scattered light [40] and second, due to potential applications of the flat bands inside ERs for active light manipulation [7] and as alternative to band edge distributed feedback lasing [55-57].

Here we provide a semianalytical yet fully electrodynamic analysis of plasmonic honeycomb arrays with spatially dependent gain. We use a lattice Green's-function method to examine the effective lattice polarizability, which includes all near-field and far-field electrodynamic dipole-dipole interactions up to all orders of scattering [58]. This effective polarizability contains both the band structure and the optical scattering response of such non-Hermitian metasurfaces, linking ERs in the band structure to a far-field chiroptical polarization response.

We consider lattice geometries derived from the basic honeycomb lattice presented in Fig. 1, with different magnitudes of gain in sublattices $A$ and $B$ providing either partial or complete compensation of plasmonic losses. In this scheme, the metasurface building blocks consist of plasmonic cores 


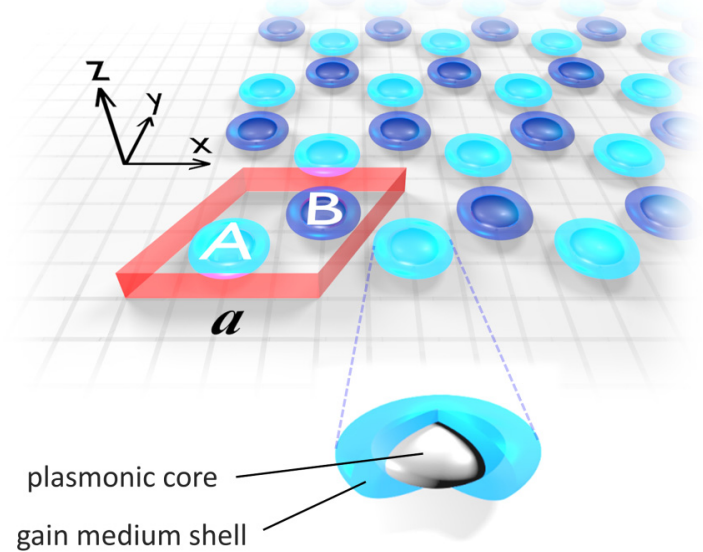

FIG. 1. Non-Hermitian metasurface made of nanoparticles with a plasmonic core and gain medium shell, which serves as a computationally feasible approximation of the usual experimental geometry of a plasmonic nanoparticle array covered by a continuous gain medium layer. The red polygon highlights the unit cell, with lattice pitch $a=160 \mathrm{~nm}$. Nanoparticles in sublattice $A$ (light blue) are surrounded by shells with a larger gain coefficient $\left(g_{A}\right)$ compared to those in sublattice $B$ ( $g_{B}$, dark blue). The above basic honeycomb geometry will be used as a starting point for modifications leading to band folding presented further in this article.

surrounded by gain medium shells embedded in a uniform passive dielectric medium. Such a geometry is not practical in terms of fabrication; however, it is straightforward to implement in computations. We use it as an approximation of the traditional geometry of plasmon lattice lasers, i.e., plasmonic nanoantenna arrays covered by continuous gain-doped films, which are, by contrast, easy to fabricate but challenging to model.

By properly modifying the basic honeycomb geometry to enable band folding, we achieve nonunitary circular dichroism, i.e., a greater-than-one differential response to the handedness of circular polarization (normalized by incident light intensity). More importantly, the origin of this chiroptical response is of nontrivial nature; it is not just ordinary pseudochirality accompanied by amplification, but it originates from the interaction of incoming light with spin-polarized flat bands inside ERs, induced solely by the gain-loss pattern and therefore regarded as a non-Hermitian counterpart of valley pseudospin [59-65].

Although the intrinsic pseudospins of subsequent eigenstates can be retrieved also in a simple tight-binding Hamiltonian model extended to vectorial form (as we show in Sec. II), we note that the rigorous Green's-function method used to obtain the main results of this paper (Secs. III and IV) is fundamentally not a tight-binding method. The Green'sfunction method is a fully electrodynamic multiple scattering method, which is neither limited to nearest-neighbor interactions nor limited to the quasistatic approximation. This is a crucial difference, as quasistatic approximations of plasmonics have no consistent optical theorem, i.e., no consistent description of extinction and far-field scattering [66,67]. Thus, prediction of the lattice far-field chiroptical response requires a rigorous electromagnetic modeling that includes the effect of gain and loss on the polarizability of plasmonic scatterers. This ingredient is also essential in predicting the band folding efficiency, which determines the visibility of scattering signatures of ERs. Our findings are of fundamental and practical importance, as they demonstrate a different property of a widely explored system and they offer different perspectives on dynamically controlled polarization-converting metasurfaces and a spin-polarized version of Dirac point/exceptional ring lasers [68-71].

This paper is organized as follows. In Sec. II we present a minimal model based on the vectorial tight-binding Hamiltonian (Sec. II A), which we use to predict the pseudospins of eigenstates in both Hermitian and non-Hermitian honeycomb lattices (Sec. II B) and to reveal their mutual connections (Sec. II C). In Sec. III we briefly review a rigorous approach based on effective polarizability and the lattice Green's function to investigate non-Hermitian collective plasmonic modes in honeycomb metasurfaces with gain and loss (Sec. III A) and we use this approach to demonstrate net ellipticity and net gain in the flat bands inside ERs (Sec. III B). In Sec. IV we present a method for calculating the far-field scattering observables (Sec. IV A) and we use this method to demonstrate the effects of band folding upon distortion of lattice geometry (Sec. IV B), accompanied by the pseudochiral response at ERs (Sec. IV C). Finally, in Sec. V we provide a brief summary and outlook, discussing the feasibility of experimental realization of metasurfaces with patterned gain (Sec. V A) and their potential in developing new kinds of lasers and active photonic elements for polarization control (Sec. V B).

\section{PSEUDOSPINS INSIDE EXCEPTIONAL RINGS IN THE MINIMAL MODEL}

\section{A. Vectorial tight-binding model}

We first introduce a minimal toy model that results in the emergence of pseudospins inside ERs within the tight-binding approximation. This is to provide a qualitative understanding of the results of the full lattice Green's-function model presented in Sec. III. To obtain the tight-binding picture we approximate the multiple scattering between plasmonic antennas by the eigenvalue problem $\mathbf{H} \boldsymbol{\psi}_{n}=\omega_{n} \boldsymbol{\psi}_{n}$, where $\boldsymbol{\psi}_{n}$ and $\omega_{n}$ are the eigenfunctions and eigenvalues, respectively, and $\mathbf{H}$ is the tight-binding lattice Hamiltonian. The honeycomb lattice consists of two primitive sublattices, which we label $A$ and $B$. We further assume that the wave function at each lattice site is a two-component vector associated with the in-plane Cartesian directions $x$ and $y$. Hence, the eigenvectors of a minimal honeycomb unit cell are $\psi_{n}=\left(\psi_{A x}, \psi_{A y}, \psi_{B x}, \psi_{B y}\right)_{n}^{T}$ and have well-defined pseudospins

$$
s_{n}=-i\left(\begin{array}{l}
\psi_{A x} \\
\psi_{A y}
\end{array}\right)_{n} \times\left(\begin{array}{l}
\psi_{A x} \\
\psi_{A y}
\end{array}\right)_{n}^{*}-i\left(\begin{array}{l}
\psi_{B x} \\
\psi_{B y}
\end{array}\right)_{n} \times\left(\begin{array}{l}
\psi_{B x} \\
\psi_{B y}
\end{array}\right)_{n}^{*} .
$$

If the nanoparticles are isotropic in the $x y$ plane, the Hamiltonian can be expressed as

$$
\mathbf{H}=\left(\begin{array}{cc}
\mathbb{I}\left(\omega_{A}+i \gamma_{A}\right) & \boldsymbol{\Omega}_{A B} \\
\boldsymbol{\Omega}_{A B}^{* T} & \mathbb{I}\left(\omega_{B}+i \gamma_{B}\right)
\end{array}\right),
$$

where $\mathbb{I}$ is the $2 \times 2$ identity operator, $\omega_{A}+i \gamma_{A}$ and $\omega_{B}+i \gamma_{B}$ are the complex-valued resonance frequencies of individual 
lattice sites in subsequent sublattices, and $\boldsymbol{\Omega}_{A B}$ is the coupling term

$$
\boldsymbol{\Omega}_{A B}=\Omega \sum_{m}^{3} \exp \left\{i \mathbf{k}_{\|} \cdot \mathbf{r}_{m}\right\} \mathbf{G}\left\{\mathbf{r}_{m}\right\},
$$

where $\mathbf{k}_{\|}$is the in-plane momentum, $\mathbf{r}_{m}=\mathbf{r}_{B, m}-\mathbf{r}_{A}$ are vectors connecting the nearest neighbors, $\Omega$ determines the coupling strength, and $\mathbf{G}\{\mathbf{r}\}$ is a normalized $2 \times 2$ matrix which encodes the symmetry of interactions. We assume that $\mathbf{G}\{\mathbf{r}\}$ is determined by the electric point dipole Green's function approximated to the near field and without retardation:

$$
\mathbf{G}\{\mathbf{r}\} \propto\left(\mathbb{I}+\frac{1}{k^{2}} \nabla \otimes \nabla\right) \frac{e^{i k|\mathbf{r}|}}{|\mathbf{r}|} \approx-\mathbb{I}+\frac{3 \mathbf{r} \otimes \mathbf{r}}{|\mathbf{r}|^{2}} .
$$

\section{B. Pseudospin texture in the momentum space}

Figure 2 shows pseudospin textures, visualized here in blue and red, that emerge at the Dirac points upon both Hermitian and non-Hermitian perturbation. Due to the realeigenfrequency degeneracies in the $\mathcal{P} \mathcal{T}$-broken phases inside ERs [Fig. 2(a)], there is no clear imbalance between the opposite pseudospins of gainy and lossy modes [Fig. 2(b)], so they seemingly balance each other. One could expect to observe these pseudospins only if one of the degenerate modes is selectively excited or has a dominant contribution to the scattered field. Such a scenario could be realized if the non-Hermitian honeycomb particle array is brought to lasing, leading to mode competition and eventual gain saturation by the gainy mode. However, within the tight-binding approximation it is impossible to go beyond the simple eigenfrequency analysis presented here, i.e., to optical response. The reason is that consistent scattering quantities require a rigorous polarizability and lattice Green's-function method with self-consistent account of radiation damping and all terms in the Green's function. We present such a model that takes into account the influence of gain and loss on the spectral bandwidth and on the magnitude of polarizability at resonance in Sec. III.

In comparison to pseudospin textures in non-Hermitian lattices, Fig. 2(c) shows the unperturbed Dirac cones of a Hermitian honeycomb lattice, in which both sublattices are resonant at the same frequency and with the same damping factor. Figure 2(d) shows the effect of detuning of resonance frequencies of two sublattices. The band edges (valleys) of newly formed band gaps are dressed with pseudospins, with opposite handedness at the upper and lower band edges [72]. In the next section, by tuning the model parameters, we will reveal the connection between spin-polarized valleys and ERs.

\section{Encircling one of the exceptional points}

In Fig. 3 we encircle one of the EPs that belongs to the ER, following a closed trajectory in parameter space (see the figure caption for a detailed description), where one parameter is $k_{x}$ and the other is the detuning of resonance frequencies of two sublattices $\left(\Delta \omega=\omega_{B}-\omega_{A}\right)$. After completing the loop, the eigenstates are interchanged, revealing the Möbius-strip topology associated with each EP that belongs to an ER [52]. Such an isolated EP resulting from coalescence of eigenstates (a)

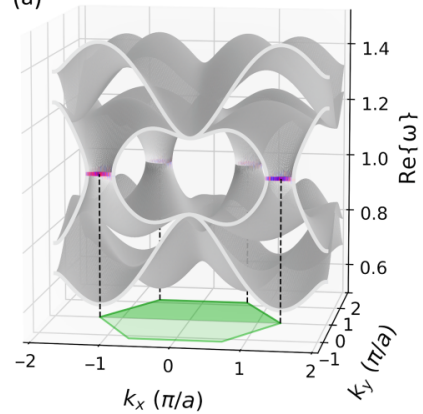

(c)

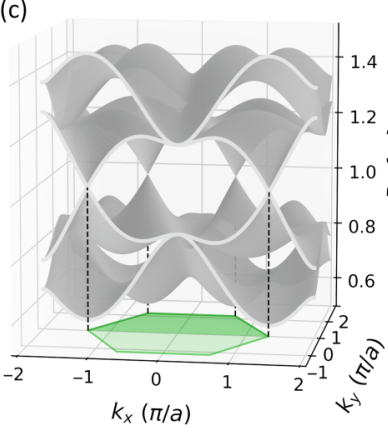

(b)

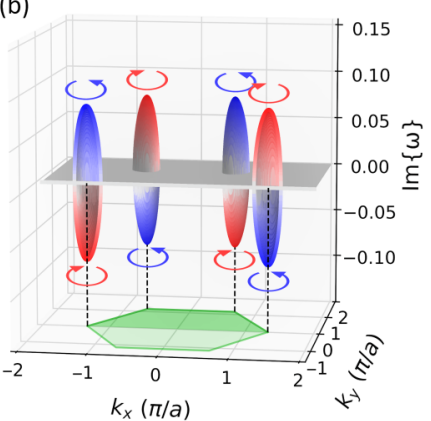

(d)
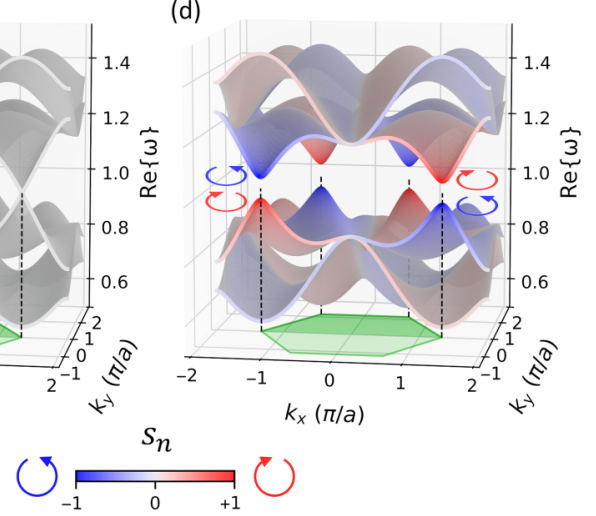

FIG. 2. Band structures obtained by diagonalization of the tightbinding Hamiltonian from Eq. (2) with coupling strength $\Omega=0.23$ and coupling symmetry defined by Eq. (4). The green hexagon at the bottom of each plot indicates the first Brillouin zone and its darker area corresponds to the part that is included in the $\mathbf{k}$ range of eigenvalue plots. The pseudospin of each eigenmode is encoded in the blue-gray-red color scale. (a) and (b) Plots of the non-Hermitian counterpart of the valley pseudospin effect caused by the detuning of imaginary resonance frequencies $\left(\omega_{A}=\omega_{B}=1\right.$ and $\left.\gamma_{B}-\gamma_{A}=0.16\right)$. This leads to breaking of $\mathcal{P} \mathcal{T}$ symmetry at the Dirac points, preserving their degeneracy in the real eigenfrequencies (a), but splitting the imaginary eigenfrequencies (b) and dressing them with pseudospins. For comparison, we show the band structures of a Hermitian honeycomb lattice $\left(\omega_{A}=\omega_{B}=1\right.$ and $\left.\gamma_{A}=\gamma_{B}=0\right)$, with two bands forming Dirac cones (c) and the band gap opening upon detuning of resonance frequencies between two sublattices $\left(\omega_{B}-\omega_{A}=0.08\right)$, giving rise to valley pseudospins (d). In these two plots (c) and (d), the imaginary eigenfrequency landscape is completely flat and therefore it is not shown.

$m$ and $n$ can be described by a topological invariant $v_{m n}$ defined as

$$
v_{m n}(\Gamma)=-\frac{1}{2 \pi} \oint_{\Gamma} \nabla_{\mathbf{s}} \arg \left[\omega_{m}(\mathbf{s})-\omega_{n}(\mathbf{s})\right] \cdot d \mathbf{s},
$$

where $\Gamma$ is a closed trajectory in some two-dimensional parameter space $\mathbf{s}$, in our case $\mathbf{s}=\left(k_{x}, \Delta \omega\right)$. The above invariant is a modified version of vorticity defined for isolated EPs in momentum space $\mathbf{s} \equiv \mathbf{k}=\left(k_{x}, k_{y}\right)$ [39]. It follows from Eq. (5) and Fig. 3 that each EP of an ER can be described by a fractional topological charge $v_{m n}= \pm \frac{1}{2}$ in the parameter space $\mathbf{s}=\left(k_{x}, \Delta \omega\right)$, where the plus or minus sign is fixed for a given ER. A remarkable observation is that the eigenstates preserve their pseudospins along the entire trajectory, except the point of band gap closing in the middle between points $S$ 


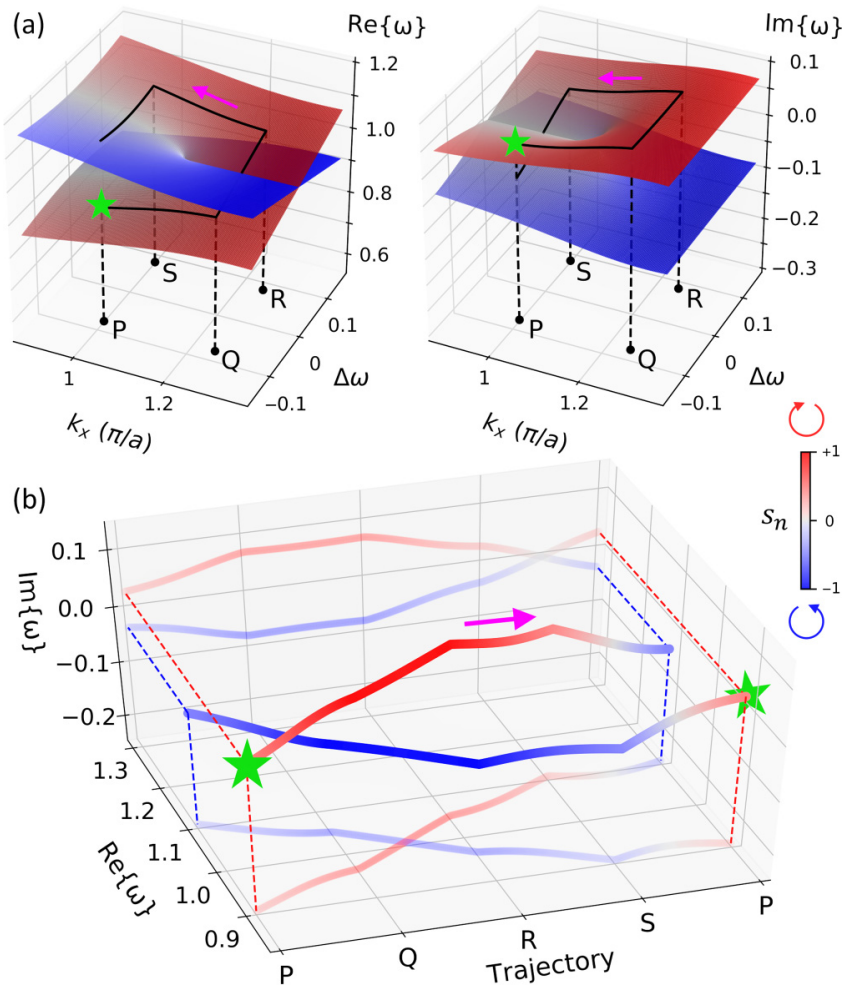

FIG. 3. (a) Complex eigenfrequencies (surface plots) and pseudospins of their corresponding eigenvectors (blue-gray-red color scale) in $k_{x}-\Delta \omega$ parameter space (where $\Delta \omega=\omega_{B}-\omega_{A}$ is the detuning of resonance frequencies of two sublattices) surrounding one of the EPs from an ER, obtained from Eq. (2) with gain-loss contrast $\gamma_{B}-\gamma_{A}=0.16$. The black solid line shows an example closed trajectory in this parameter space, leading to an interchange of eigenstates upon encircling the EP $[25,26,33]$. The trajectory starts from the lower-frequency state (green star) at point $P,\left(k_{x}=\pi / a, \Delta \omega=\right.$ $-0.08)$; goes through points $Q,\left(k_{x}=5 \pi / 4 a, \Delta \omega=-0.08\right) ; R$, $\left(k_{x}=5 \pi / 4 a, \Delta \omega=0.08\right)$; and $S,\left(k_{x}=\pi / a, \Delta \omega=0.08\right)$ (following the pink arrow); and then it is back to $P$, ending in the higher-frequency state. (b) Evolution of the two eigenfrequencies in the complex plane upon encircling the EP, following the trajectory presented in (a).

and $P$, when the sublattice detuning is swept from positive to negative at $k_{x}=\pi / a$, i.e., outside the ER. In contrast, band gap closing between points $Q$ and $R$ at $k_{x}=5 \pi / 4 a$, i.e., inside the ER, leads to reversing the real-frequency ordering of eigenstates without changing their pseudospins. This allows us to consider pseudospins inside $K$-point ERs as nonHermitian counterparts of the $K$-point valley pseudospins.

\section{SPIN-POLARIZED PLASMONIC LATTICE MODES INSIDE EXCEPTIONAL RINGS}

We will now turn to describing the pseudochiral properties of photonic modes associated with ERs in a concrete physical system, without the crude approximations of the tight-binding method. The system at hand is a honeycomb array of coreshell plasmonic nanoparticles with nonuniform gain, with basic structure presented in Fig. 1. We start this section by briefly introducing the formalism behind the optical response of periodic metasurfaces, which is based on Ewald summation techniques and the full electromagnetic dyadic Green's function.

\section{A. Theoretical model: Effective polarizability and lattice Green's function}

Our calculations are based on a lattice Green's-function approach, in which metasurface scatterers are treated as electrodynamic point dipole scatterers and interactions between all scatterers are accounted for, with full inclusion of retardation effects and to all orders of multiple scattering. The main approximation is that higher-order multipole moments of the scatterers are ignored. This approximation aside, the method is well known to give an accurate and self-consistent description of the multiple scattering response of clusters, with a closed energy balance (in the absence of gain), also for scatterers with cross sections as large as the unitary limit $\left(3 / 2 \pi \lambda^{2}\right.$, the largest possible scattering cross section for any dipolar scatterer). A general multiple scattering problem for an ensemble of $N$ electric point dipole scatterers can be formulated as $[73,74]$

$$
\mathbf{p}_{i}=\boldsymbol{\alpha}_{i}\left[\mathbf{E}_{\mathrm{inc}}\left(\mathbf{r}_{i}\right)+\sum_{j=1, i \neq j}^{N} \mathbf{G}^{0}\left(\omega, \mathbf{r}_{i}-\mathbf{r}_{j}\right) \mathbf{p}_{j}\right],
$$

where $\mathbf{p}_{i}$ are the dipoles induced at subsequent scatters located at positions $\mathbf{r}_{i}$, with polarizabilities $\boldsymbol{\alpha}_{i}$, and $\mathbf{G}^{0}$ is the dyadic Green's function

$$
\mathbf{G}^{0}\left(\omega, \mathbf{r}-\mathbf{r}^{\prime}\right)=\left(\mathbb{I} k^{2}+\nabla \otimes \nabla\right) \frac{e^{i k\left|\mathbf{r}-\mathbf{r}^{\prime}\right|}}{\left|\mathbf{r}-\mathbf{r}^{\prime}\right|},
$$

with $k=\omega n / c$ denoting the wave number in the embedding medium and $n$ its refractive index. For lattices having more than one scatterer per unit cell, the position vectors can be defined as

$$
\mathbf{r}_{i} \equiv \mathbf{r}_{l m n}=\mathbf{r}_{l}+\mathbf{R}_{m n},
$$

where $l$ enumerates the scatterers inside the unit cell at the origin ( $m, n=0)$ and $\mathbf{R}_{m n}=m \mathbf{a}_{1}+n \mathbf{a}_{2}$ are the lattice vectors defined in terms of basis vectors $\mathbf{a}_{1}$ and $\mathbf{a}_{2}$. For the honeycomb lattice, we set $\mathbf{a}_{1}=a(1,0,0)^{T}, \mathbf{a}_{2}=a\left(\frac{1}{2}, \sqrt{3} / 2,0\right)^{T}$, and $\mathbf{r}_{B}-\mathbf{r}_{A}=a\left(\frac{1}{2}, \sqrt{3} / 6,0\right)^{T}$. Under plane-wave illumination

$$
\mathbf{E}_{\text {inc }}\left(\mathbf{r}_{i}\right)=\mathbf{E}_{0} e^{i \mathbf{k} \cdot \mathbf{r}_{i}},
$$

Bloch's theorem allows us to express the dipole moments as

$$
\mathbf{p}_{l m n}=\mathbf{p}_{l} e^{i \mathbf{k}_{\| \cdot} \cdot \mathbf{R}_{m n}},
$$

where $\mathbf{p}_{l}$ can be expressed as

$$
\mathbf{p}_{l}=\boldsymbol{\alpha}_{l}\left[\mathbf{E}_{0} e^{i \mathbf{k} \cdot \mathbf{r}_{l}}+\mathcal{G}^{\neq} \mathbf{p}_{l}+\sum_{l^{\prime}=1, l^{\prime} \neq l}^{N} \mathcal{G}_{l l^{\prime}} \mathbf{p}_{l^{\prime}}\right],
$$

where $\mathcal{G}^{\neq}$determines the coupling between scatterers of the same sublattice across different unit cells

$$
\mathcal{G}^{\neq}=\sum_{m \neq 0, n \neq 0} \mathbf{G}^{0}\left(-\mathbf{R}_{m n}\right) e^{i \mathbf{k}_{\|} \cdot \mathbf{R}_{m n}}
$$


and $\mathcal{G}_{l l^{\prime}}$ is responsible for the coupling between scatterers in different sublattices [75]

$$
\mathcal{G}_{l l^{\prime}}=\sum_{m, n} \mathbf{G}^{0}\left(\mathbf{r}_{l}-\mathbf{r}_{l^{\prime}}-\mathbf{R}_{m n}\right) e^{i \mathbf{k}_{\|} \cdot \mathbf{R}_{m n}} .
$$

Technical details on calculating the above lattice sums are given in Appendix A.

The solution of Eq. (11) is

$$
\mathbf{p}\left(\omega, \mathbf{k}_{\|}\right)=\left(\begin{array}{c}
\mathbf{p}_{1}\left(\omega, \mathbf{k}_{\|}\right) \\
\vdots \\
\mathbf{p}_{l}\left(\omega, \mathbf{k}_{\|}\right) \\
\vdots \\
\mathbf{p}_{N}\left(\omega, \mathbf{k}_{\|}\right)
\end{array}\right)=\boldsymbol{\alpha}_{\mathrm{eff}}\left(\omega, \mathbf{k}_{\|}\right)\left(\begin{array}{c}
\mathbf{E}_{0} e^{i \mathbf{k} \cdot \mathbf{r}_{1}} \\
\vdots \\
\mathbf{E}_{0} e^{i \mathbf{k} \cdot \mathbf{r}_{l}} \\
\vdots \\
\mathbf{E}_{0} e^{\mathbf{i k \cdot \mathbf { r } _ { N }}}
\end{array}\right),
$$

where $\boldsymbol{\alpha}_{\text {eff }}$ is the effective lattice polarizability [52,58,75-79] governed by

$$
\boldsymbol{\alpha}_{\text {eff }}^{-1}\left(\omega, \mathbf{k}_{\|}\right)=\boldsymbol{\alpha}^{-1}(\omega)-\mathbf{G}_{\text {latt }}\left(\omega, \mathbf{k}_{\|}\right),
$$

where $\boldsymbol{\alpha}$ is a block-diagonal matrix

$$
\boldsymbol{\alpha}(\omega) \equiv\left(\begin{array}{ccccc}
\boldsymbol{\alpha}_{1} & \cdots & \mathbf{0} & \cdots & \mathbf{0} \\
\vdots & \ddots & \vdots & & \vdots \\
\mathbf{0} & \cdots & \boldsymbol{\alpha}_{l} & \cdots & \mathbf{0} \\
\vdots & & \vdots & \ddots & \vdots \\
\mathbf{0} & \cdots & \mathbf{0} & \cdots & \boldsymbol{\alpha}_{N}
\end{array}\right)
$$

containing electric polarizabilities of subsequent scatterers in one unit cell and $\mathbf{G}_{\text {latt }}\left(\omega, \mathbf{k}_{\|}\right)$is the lattice Green's function

$$
\mathbf{G}_{\text {latt }}\left(\omega, \mathbf{k}_{\|}\right) \equiv\left(\begin{array}{ccccc}
\mathcal{G}^{\neq} & \cdots & \mathcal{G}_{1 l} & \cdots & \mathcal{G}_{1 N} \\
\vdots & \ddots & \vdots & & \vdots \\
\mathcal{G}_{l 1} & \cdots & \mathcal{G}^{\neq} & \cdots & \mathcal{G}_{l N} \\
\vdots & & \vdots & \ddots & \vdots \\
\mathcal{G}_{N 1} & \cdots & \mathcal{G}_{N l} & \cdots & \mathcal{G}^{\neq}
\end{array}\right),
$$

with diagonal blocks $\mathcal{G}^{\neq}$and off-diagonal blocks $\mathcal{G}_{l l^{\prime}}$. To be consistent with the optical theorem, the polarizabilities $\boldsymbol{\alpha}_{l}$ of individual scatterers must be corrected by a radiative damping term [80]

$$
\boldsymbol{\alpha}_{l}^{-1}(\omega)=\boldsymbol{\alpha}_{\mathrm{stat}, l}^{-1}(\omega)-\frac{2 i k^{3}}{3} \mathbb{I},
$$

where $\boldsymbol{\alpha}_{\text {stat }, l}$ are static electric point dipole polarizabilities of subsequent scatterers, which are obtained from an analytical formula for a core-shell ellipsoid [81] (see Appendix B). We consider a honeycomb lattice of pitch $a=160 \mathrm{~nm}$, made of ellipsoidal silver core/gain medium shell nanoparticles of aspect ratio $r_{x y}: r_{z}=2.431: 1$, total volume $1.25 \times 10^{-22} \mathrm{~m}^{3}$ per nanoparticle, and volume fraction of the core equal to $\left(\frac{2}{3}\right)^{3}$, yielding core radius $r_{x y} \approx 28 \mathrm{~nm}$ and lateral shell thickness approximately equal to $14 \mathrm{~nm}$. Optical constants of silver are based on Ref. [82], whereas the gain medium of the shell and the passive embedding medium both have a refractive index $n=1.5$. The shell is imbued with Lorentzian gain [83-85] with relative permittivity

$$
\varepsilon_{g}=n^{2}\left(1+g k_{g}^{-1} \frac{\gamma_{g}}{\omega_{g}-\omega+i \gamma_{g}}\right),
$$

where $k_{g}=n \omega_{g} / c$ is the wave number in the gain medium at the gain peak. We assume a gain bandwidth $\hbar \gamma_{g}=0.4 \mathrm{eV}$, spectral gain peak position $\hbar \omega_{g}=2.6 \mathrm{eV}$, and peak gain coefficients $g$ in the range $10^{3}-10^{4} \mathrm{~cm}^{-1}$, necessary to balance the imaginary part of the electric permittivity of silver, ensuring that $\boldsymbol{\alpha}_{\text {stat }}$ in Eq. (15) provides amplification upon scattering. We assume no amplification through propagation to avoid summation of complex Green's functions, although such amplification would normally contribute to $\mathbf{G}_{\text {latt }}$ (being in fact the main amplification mechanism in plasmon lattice lasers), possibly allowing much lower gain coefficients for achieving the same non-Hermitian effects.

We include gain in our model by considering nanoparticles with static polarizabilities $\boldsymbol{\alpha}_{\text {stat }}$ having a negative imaginary part, resulting from the negative imaginary part of $\varepsilon_{g}$ [Eq. (19)]. This is analogous to the case of absorbing scatterers, where $\operatorname{Im}\left\{\boldsymbol{\alpha}_{\text {stat }}\right\}$ is positive due to a positive imaginary part of permittivity of the scatterer's material. Therefore, we account for radiation damping via Eq. (18), which is known to hold for absorbing scatterers regardless of nonzero $\operatorname{Im}\left\{\boldsymbol{\alpha}_{\text {stat }}\right\}$ [80]. However, rigorous treatment of amplified scattering requires us to consider the energy balance that includes both the pump and probe, and hence it becomes dependent on a particular gain mechanism [86]. We leave aside a detailed analysis of this aspect as it is beyond the main scope of this work.

\section{B. Net gain and net ellipticity of plasmonic lattice modes inside exceptional rings}

Evaluation of $\boldsymbol{\alpha}_{\text {eff }}$ using Eq. (15) provides access to far-field scattering observables (transmission and reflection, which will be addressed in Sec. IV) and certain properties of guided modes. We focus on net extinction, defined as

$$
\sigma_{\text {net }}=\frac{4 \pi k a}{V} \sum_{j} \operatorname{Im}\left\{\alpha_{\mathrm{eff}, j}\right\}
$$

where $\alpha_{\text {eff }, j}$ are the eigenvalues of $\boldsymbol{\alpha}_{\text {eff }}, a$ is the lattice pitch, and $V$ is the total volume of the nanoparticles inside one unit cell. This definition relates to the single-particle extinction cross section $\sigma_{\mathrm{ext}}=4 \pi k \operatorname{Im}\{\alpha\} / S$ normalized to a particle's cross-sectional area $S$. Generalized to lattices, net extinction is a measure for the extinction cross section per unit cell (positive for net extinguishing and negative for net amplifying) contributed by all the lattice eigenmodes. It can be evaluated at any given point in $\mathbf{k}_{\|}-\omega$ space, also outside the light cone, and it can be separated into contributions of subsequent eigenpolarizabilities.

Figure 4(a) reveals the main features of the photonic band structure of the metasurface presented in Fig. 1, displaying the $k_{x}-\omega$ map of net extinction associated with the in-plane polarized modes (we found that the out-of-plane polarized modes are significantly blue detuned and do not couple with the in-plane modes [52], and therefore we excluded them from our model by setting $\alpha_{\text {stat, } z z}=0$ ). Four in-plane polarized modes are visible, corresponding to two dipoles per unit cell, each with two degrees of freedom $(x$ and $y)$. Within the light cone, the only visible features are the subradiant 

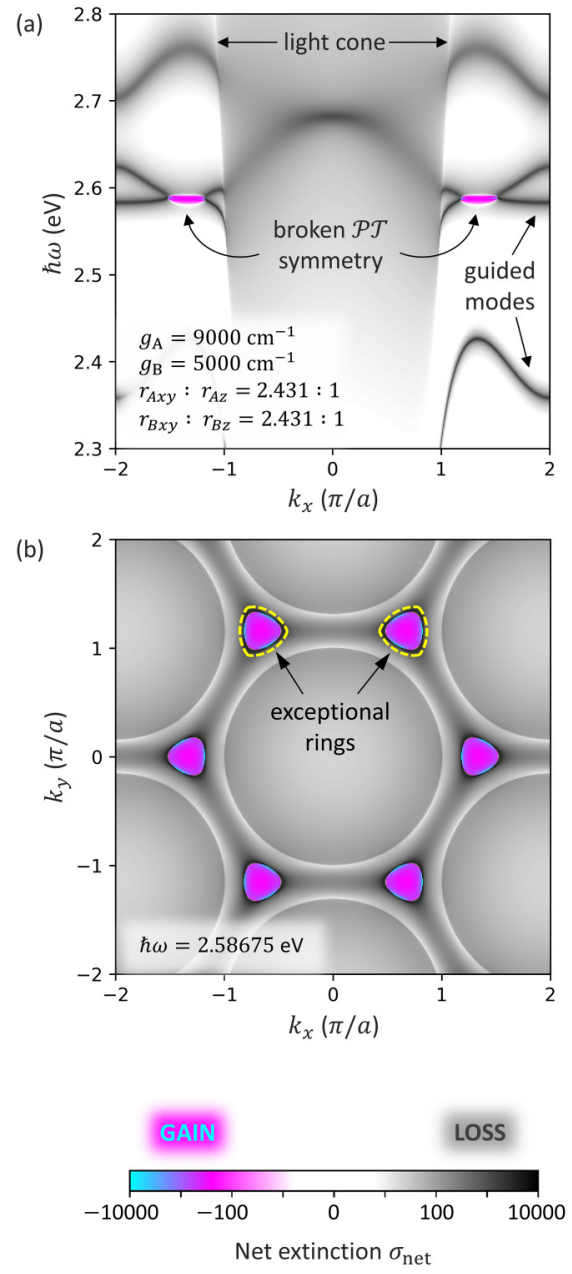

(c)

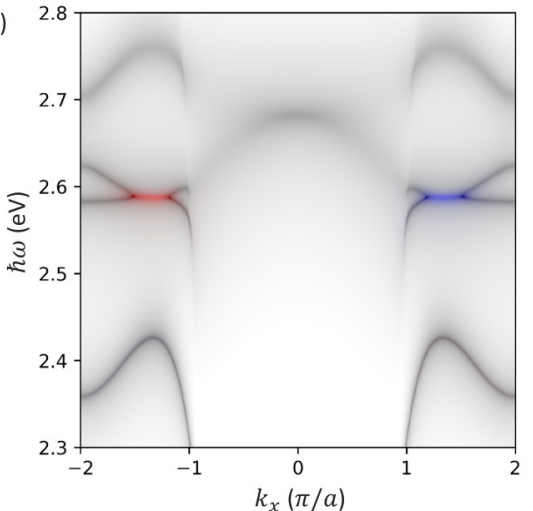

(d)

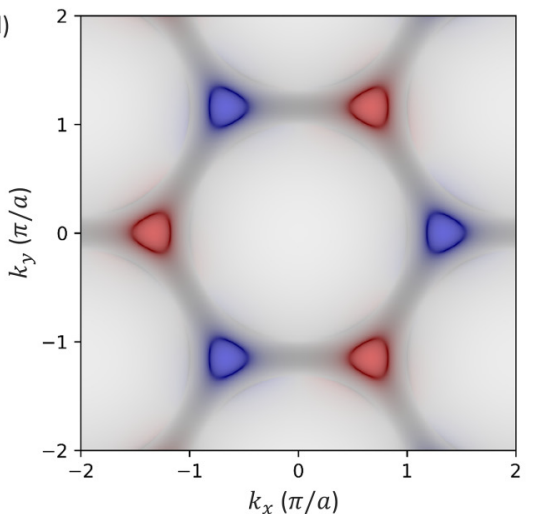

(e)

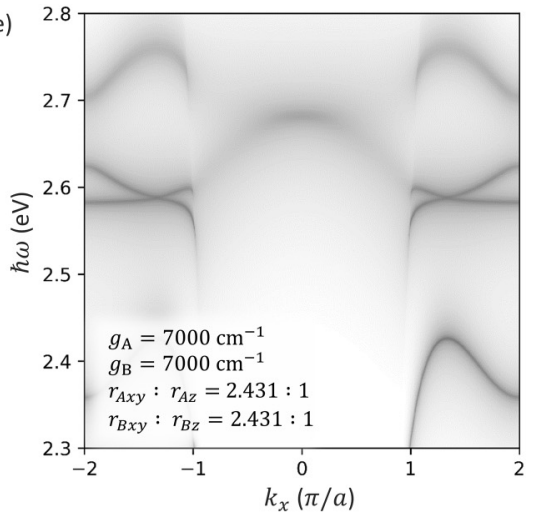

(f)

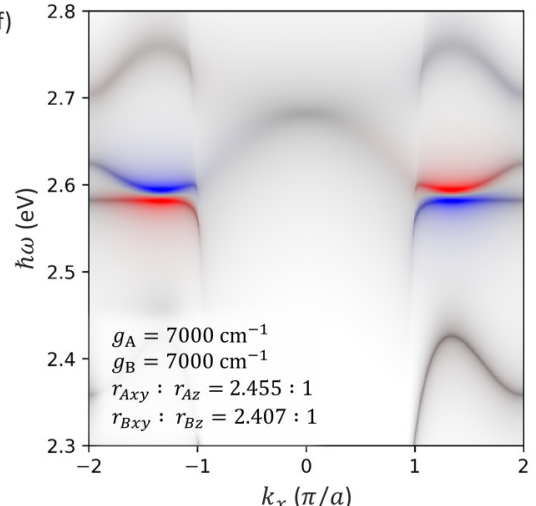

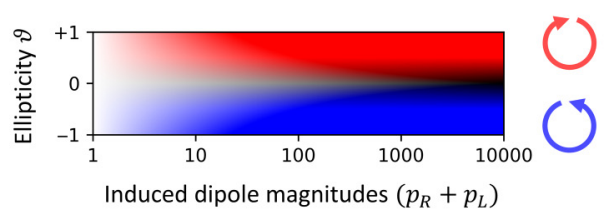

FIG. 4. (a) Frequency-momentum map of net extinction [defined in Eq. (20)] for active plasmonic metasurface schematically shown in Fig. 1, with lattice pitch $a=160 \mathrm{~nm}, g_{A}=9000 \mathrm{~cm}^{-1}$, and $g_{B}=5000 \mathrm{~cm}^{-1}$. The most important features of the photonic band structure are pointed out. (b) Crosscut of the momentum space at $\hbar \omega=2.58675 \mathrm{eV}$, capturing the flat bands with net gain inside ERs (magenta color) and highlighting the most likely location of two out of six ERs (yellow contours). (c)-(f) Frequency-momentum maps of net ellipticity (blue-gray-red scale), additionally multiplied by $\log _{10}$ of the sum of induced dipole magnitudes in order to reveal the location of photonic bands. Plots (c) and (d) are obtained for the non-Hermitian plasmonic metasurface and are analogous to (a) and (b). Plots (e) and (f) are calculated for plasmonic metasurfaces with uniform gain $g_{A}=g_{B}=7000 \mathrm{~cm}^{-1}$. Plot (e) assumes identical sublattices, whereas plot (f) introduces a detuning of their resonances by changing the aspect ratio of ellipsoids to 2.455 in sublattice $A$ (higher aspect ratio equals redshifted resonant frequency [87]) and to 2.407 in sublattice $B$ (lower aspect ratio equals blueshifted resonant frequency). Momentum-dependent ellipticities at the $K$-point ERs and valleys are clearly visible in (c), (d), and (f), while being absent in the unperturbed lattice in (e), which shows qualitative agreement with the tight-binding model presented in Sec. II and Fig. 2.

(antisymmetric) modes, whereas the superradiant (symmetric) modes provide the broad background. Beyond the light line are guided modes. Very close to the light line, these modes are very weakly guided, while towards the Brillouin zone edge they become more strongly confined. We note that the intrinsically quasistatic tight-binding model fundamentally fails to capture the superradiant/subradiant damping of the various modes, as well as the polariton anticrossing of the plasmon resonance with the light line, which gives rise to the weakly guided modes. For larger wave vectors, well beyond the light line, the modes of the full model are more akin to those of the tight-binding model $[66,67]$.

The main features of our interest are flat bands with net gain (magenta color) at the intersections of guided modes around the $K$ points, $k_{x}= \pm 4 \pi / 3 a$. These flat bands correspond to the interiors of ERs, each bordered by a pair of EPs in the momentum-space crosscut along $k_{x}$. The fact that these EPs form closed contours around $K$ points is evident from Fig. 4(b), showing the momentum-space distribution of net extinction at a fixed frequency coinciding with the central frequency of the flat bands. This plot clearly shows that each of the net gain regions associated with flat bands has a limited extent in the momentum space.

Pseudochiral properties of these modes can be revealed by net ellipticity, defined as

$$
\vartheta=\frac{p_{R}-p_{L}}{p_{R}+p_{L}}
$$



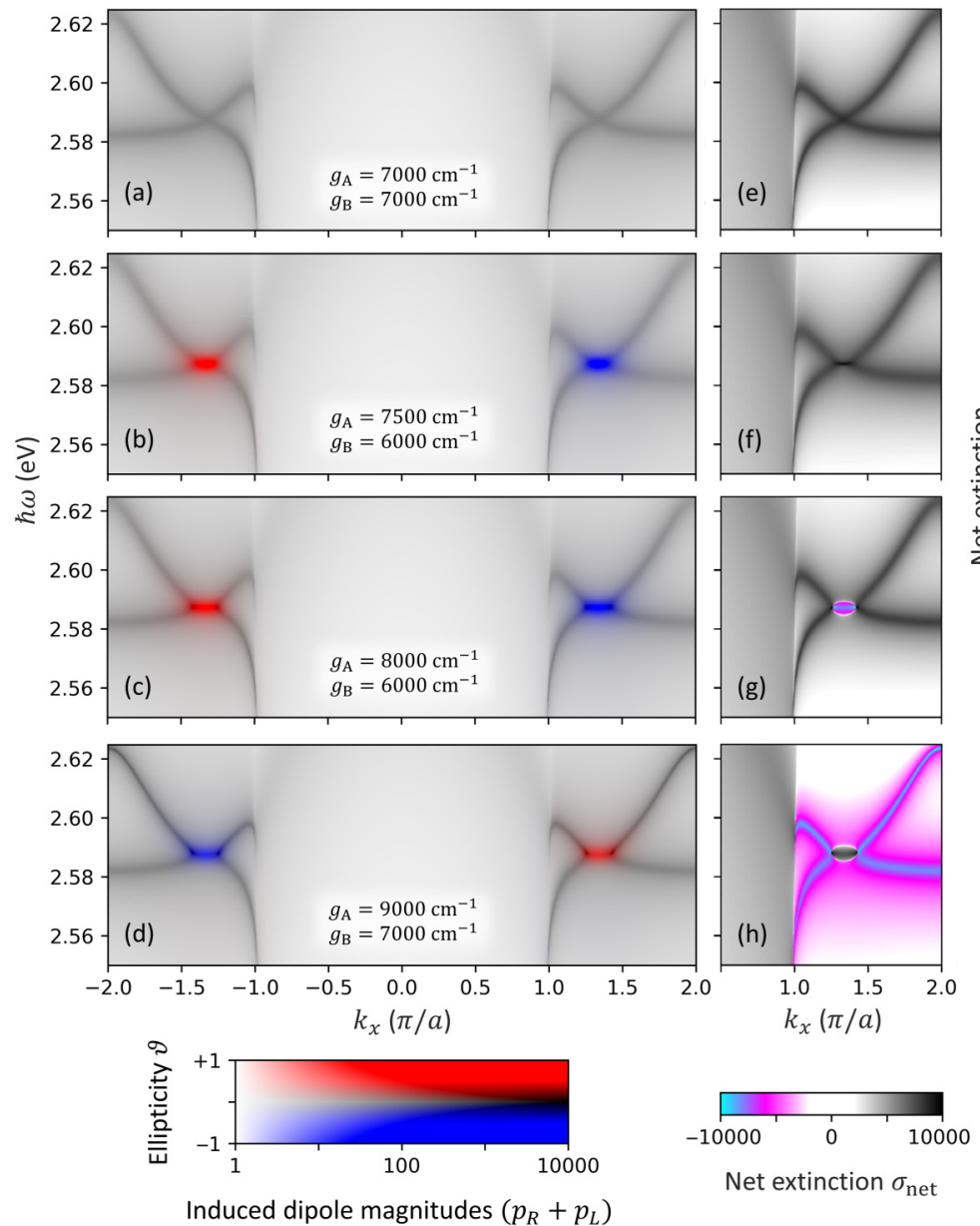
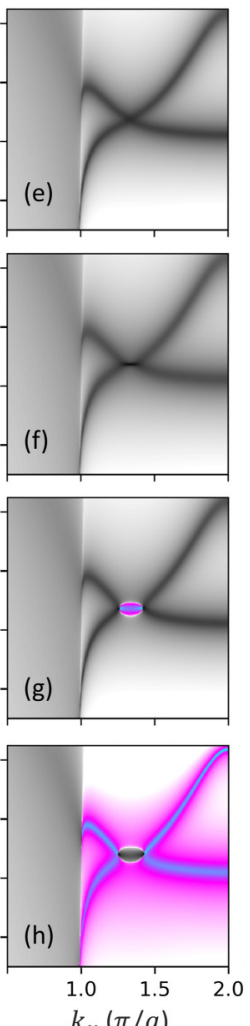

$k_{x}(\pi / a)$

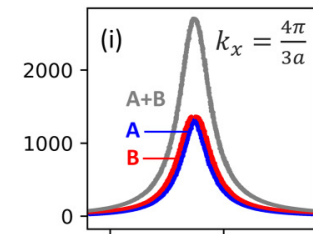

(m)
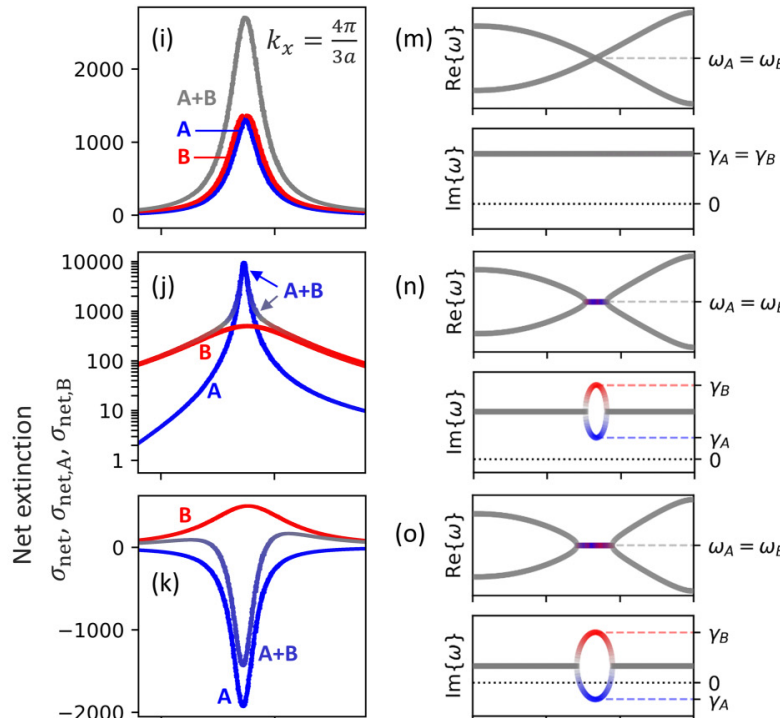

(n)
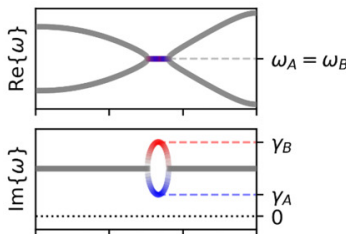

(o)
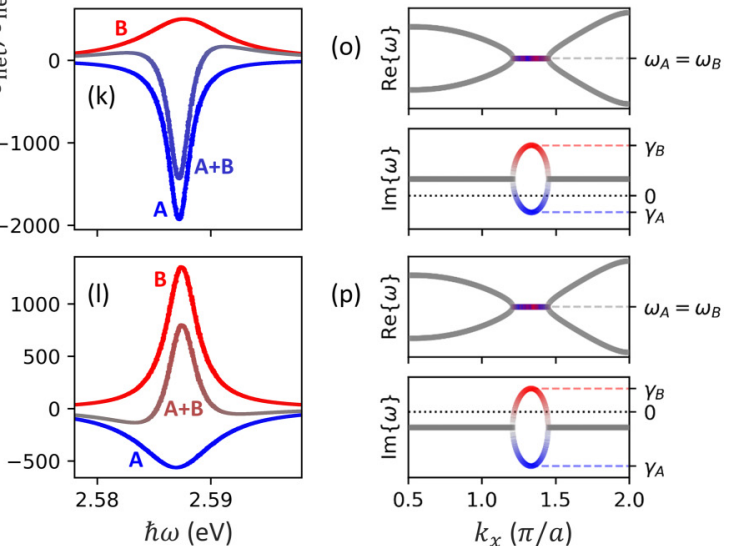

(p)
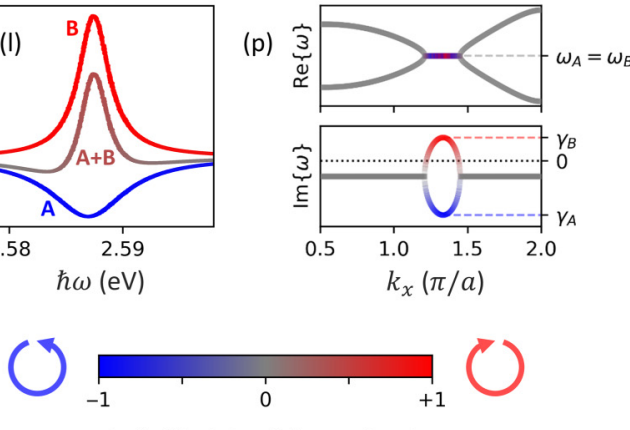

Optical ellipticity $\vartheta /$ Pseudospin $s_{n}$

FIG. 5. Four regimes of $\mathcal{P} \mathcal{T}$-symmetry breaking: (a), (e), (i), and (m) $\mathcal{P} \mathcal{T}$ unbroken; (b), (f), (j), and (n) $\mathcal{P} \mathcal{T}$ broken below the lasing threshold; and $\mathcal{P} \mathcal{T}$ broken above the lasing threshold with (c), (g), (k), and (o) net gain and (d), (h), (l), and (p) net loss inside ERs. Subsequent groups of panels show $k_{x}-\omega$ maps of (a)-(d) net ellipticity [analogous to Figs. 4(c), 4(e), and 4(f)] and (e)-(h) net extinction, accompanied by (i)-(l) spectral dependence of the contributions to net extinction associated with sublattices $A$ and $B$, and their sum, at a fixed $k_{x}=4 \pi / 3 a$. Optical ellipticity [defined in Eq. (21)] is encoded in the blue-gray-red color scale. (m)-(p) Corresponding traces of complex eigenfrequencies with pseudospins encoded in the blue-gray-red color scale, obtained from a tight-binding Hamiltonian, with lattice sites resonant at $\omega_{A}+i \gamma_{A}$ and $\omega_{B}+i \gamma_{B}$ (see Sec. II).

where $p_{R}$ and $p_{L}$ represent the lattice response to spinpolarized vector fields $\mathbf{E}_{R}$ and $\mathbf{E}_{L}$,

$$
\begin{aligned}
& p_{R}=\frac{1}{2 V}\left[\left|\boldsymbol{\alpha}_{\mathrm{eff}}\left(\begin{array}{c}
\mathbf{E}_{R}\left(\mathbf{r}_{A}\right) \\
\mathbf{E}_{R}\left(\mathbf{r}_{B}\right)
\end{array}\right)\right|+\left|\boldsymbol{\alpha}_{\mathrm{eff}}\left(\begin{array}{c}
\mathbf{E}_{R}\left(\mathbf{r}_{A}\right) \\
-\mathbf{E}_{R}\left(\mathbf{r}_{B}\right)
\end{array}\right)\right|\right], \\
& p_{L}=\frac{1}{2 V}\left[\left|\boldsymbol{\alpha}_{\mathrm{eff}}\left(\begin{array}{c}
\mathbf{E}_{L}\left(\mathbf{r}_{A}\right) \\
\mathbf{E}_{L}\left(\mathbf{r}_{B}\right)
\end{array}\right)\right|+\left|\boldsymbol{\alpha}_{\mathrm{eff}}\left(\begin{array}{c}
\mathbf{E}_{L}\left(\mathbf{r}_{A}\right) \\
-\mathbf{E}_{L}\left(\mathbf{r}_{B}\right)
\end{array}\right)\right|\right], \\
& \mathbf{E}_{R}(\mathbf{r})=e^{i \mathbf{k}_{\|} \cdot \mathbf{r}}(1,-i, 0)^{T}, \\
& \mathbf{E}_{L}(\mathbf{r})=e^{i \mathbf{k}_{\|} \cdot \mathbf{r}}(1, i, 0)^{T} .
\end{aligned}
$$

In the above relations, $p_{R}$ and $p_{L}$ are obtained by averaging the magnitudes of the dipole moments induced under symmetric and antisymmetric excitations, which is relevant for a bipartite lattice [52]. Strong ellipticities of the bands indicate chiroptical spin-polarized properties of the lattice modes. Figures 4(c), 4(d), and 4(f) clearly show that momentumdependent nonzero net ellipticity is unambiguously present in both non-Hermitian and gapped Hermitian cases. In the non-Hermitian honeycomb metasurface, the most pronounced ellipticity appears inside ERs [Figs. 4(c) and 4(d)], whereas in the Hermitian metasurface with sublattice detuning, significant ellipticity can be observed at the valleys [Fig. 4(f)], with good qualitative agreement with the tight-binding results in Fig. 2(d). At the same time, the unperturbed Hermitian lattice does not show net ellipticity at any point [Fig. 4(e)], in agreement with the tight-binding model predictions in Fig. 2(c).

Figures 5(a)-5(1) demonstrate how the modes beyond the light line evolve upon increasing gain-loss contrast, in qualitative comparison to complex eigenfrequencies from a tight-binding model [Figs. 5(m)-5(p)]. While for uniformly distributed gain [Figs. 5(a), 5(e), and 5(m)] the intersecting bands form Dirac points, a nonuniform distribution of gain destroys the degeneracy in $\operatorname{Im}\{\omega\}$, giving rise to flat bands in $\operatorname{Re}\{\omega\}$ [Figs. 5(b)-5(d), 5(f)-5(h), and 5(n)-5(p)], a signature of $\mathcal{P} \mathcal{T}$-symmetry breaking. Net gain is acquired by the flat bands [Figs. 5(g) and 5(k)] when one of the imaginary eigenvalues crosses zero [Fig. 5(o)]. After it crosses and goes into more deeply negative $\operatorname{Im}\{\omega\}$ [Fig. 5(p)], net loss results [Figs. 5(h) and 5(l)]. In between the scenarios presented in 

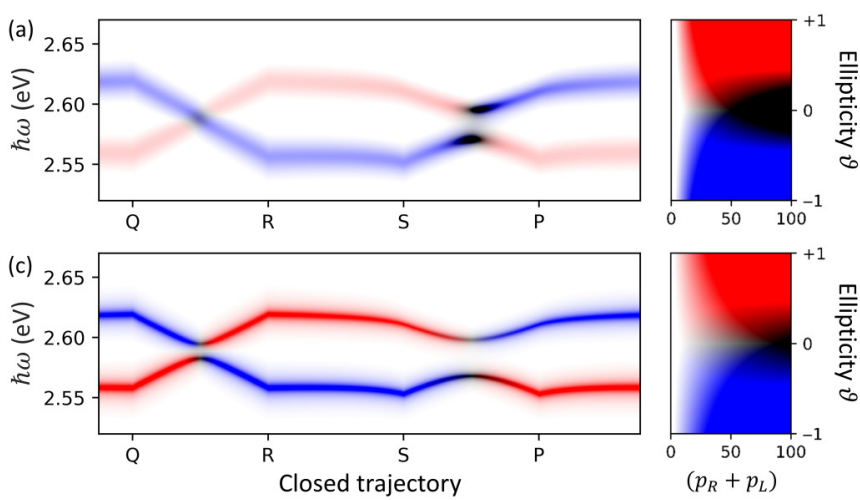

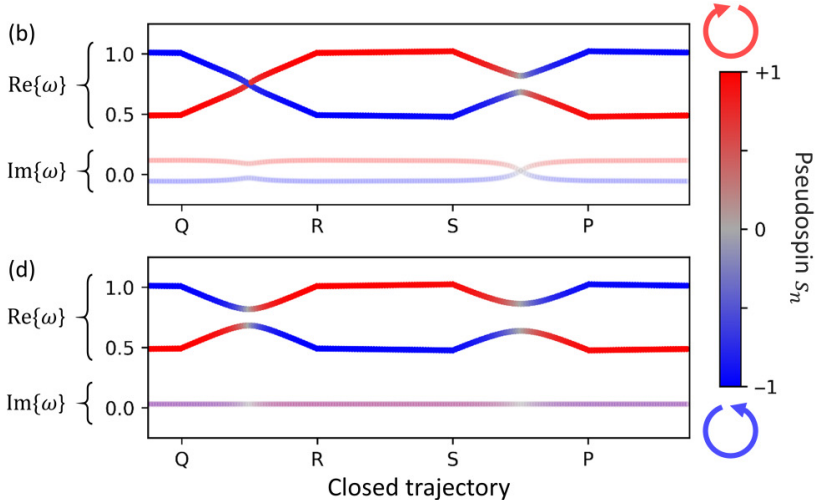

FIG. 6. (a) Evolution of photonic bands and their ellipticities upon encircling one of the EPs from an ER, following a closed trajectory analogous to that in Fig. 3, with subsequent points corresponding to different values of $k_{x}$ and aspect ratios $\mathcal{A}=r_{x y} / r_{z}$ of ellipsoidal nanoparticles: $Q, k_{x}=1.16 \pi / a, \mathcal{A}_{A}=2.309$, and $\mathcal{A}_{B}=2.553 ; R, k_{x}=1.16 \pi / a, \mathcal{A}_{A}=2.553$, and $\mathcal{A}_{B}=2.309 ; S, k_{x}=1.02 \pi / a, \mathcal{A}_{A}=2.553$, and $\mathcal{A}_{B}=2.309$; and $P, k_{x}=1.02 \pi / a, \mathcal{A}_{A}=2.309$, and $\mathcal{A}_{B}=2.553$. The gain-loss contrast is strongly increased $\left(g_{A}=11000 \mathrm{~cm}^{-1}\right.$ and $g_{B}=3000 \mathrm{~cm}^{-1}$ ) in order to expand the ER along $k_{x}$ such that one of its EPs is moved to $k_{x}<1.16 \pi / a$ and can be encircled by the above trajectory. (b) The results of effective lattice polarizability calculations show excellent qualitative agreement with the rough tight-binding Hamiltonian analog, in which $\gamma_{B}-\gamma_{A}=0.18$ and the trajectory is the same in momentum space ( $k_{x}$ from $1.02 \pi / a$ to $1.16 \pi / a$ ), while $\omega_{A}$ and $\omega_{B}$ are tuned in the range from 0.5 to 1.0. (c) and (d) Evolution of bands and their ellipticities along the same trajectory as in (a) and (b) but for a Hermitian honeycomb lattice with uniformly distributed gain. As opposed to the non-Hermitian case in (a) and (b), in which the two modes interchange in the middle between $Q$ and $R$ and preserve their ellipticities, in the Hermitian case the ordering of modes along the real frequency axis remains the same: They anticross between $Q$ and $R$ and they swap their ellipticities.

Figs. 5(b), 5(f), 5(j), and 5(n) and Figs. 5(c), 5(g), 5(k), and $5(\mathrm{o})$, there is a point at which the imaginary part of effective polarizability changes its sign. At resonance, this point leads to divergence of $\left|\boldsymbol{\alpha}_{\text {eff }}\right|$, which is a scattering anomaly that can be classified as a laser [85,88]. Therefore, we will refer to this point as the lasing threshold [84], although in practice the actual lasing thresholds are strongly dependent on many additional factors such as coherence ranges and gain medium dynamics $[89,90]$, which are not relevant to our simplified model that assumes infinitely periodic time-harmonic systems.

The effect of $\mathcal{P} \mathcal{T}$-symmetry breaking on net extinction and net ellipticity can be understood by analysis of the resonance linewidth at the $K$ point. Figured 5(i)-5(1) show crosscuts at $k_{x}=4 \pi / 3 a$ along the $\omega$ axis, separating the contributions of sublattices $A$ and $B$ and showing their optical ellipticity (bluegray-red scale). In Fig. 5(i), both sublattices have equal gain, which renders their eigenmodes identical in extinction and bandwidth such that their opposite ellipticities balance each other. Once sublattice $A$ receives more gain than sublattice $B$, the resonance of $A$ becomes dominant over $B$ in extinction and polarizability. As a result, net extinction and net ellipticity approach that of the dominant eigenstate [Figs. 5(j) and 5(k)]. Net ellipticity remains the same just below and just above the lasing threshold [Figs. 5(b), 5(c), 5(f), 5(g), 5(j), and 5(k)], but can be reversed by excessive gain [Figs. 5(d), 5(h), and 5(1)], which overbroadens the gainy resonance $A$. Such a scenario, as well as other scenarios with negative extinction, corresponds to unstable solutions that in reality involve complex dynamics of mode competition and gain saturation, which are not included in our frequency-domain model. Therefore, in our further considerations of the scattering response presented in Sec. IV we will focus on the regimes below the lasing threshold, similar to Figs. 5(b), 5(f), 5(j), and 5(n), returning to a brief discussion on lasing at the end of this article.
The spin polarization of $\mathcal{P} \mathcal{T}$-broken eigenstates revealed in Figs. 5(b)-5(d) and 5(j)-5(l) is in good correspondence with predictions of a tight-binding Hamiltonian model, as presented in Figs. 5(n)-5(p). However, such eigenfrequency analysis performed using a tight-binding model does not account for the magnitude and linewidth of $\boldsymbol{\alpha}_{\mathrm{eff}}$, which are the essential factors that lead to nonzero net ellipticity inside ERs in Figs. 5(b)-5(d) and 5(j)-5(1). This is because spin-polarized flat bands in the $\mathcal{P} \mathcal{T}$-broken phase inside ERs are frequency degenerate such that their ellipticities would cancel each other in the absence of imbalance offered by $\boldsymbol{\alpha}_{\text {eff }}$. This is in contrast to gapped Hermitian lattices [Figs. 2(d) and 4(f)], where spin-polarized valleys are separated in frequency, which by itself prevents the ellipticities of these modes from balancing each other.

\section{Topological structure of $\boldsymbol{K}$-point exceptional rings}

The tight-binding model is a straightforward tool for studying the connection between ERs and topological band gaps, which has been demonstrated in Fig. 3. Similar analysis can be done using $\boldsymbol{\alpha}_{\text {eff }}$, which is presented in Fig. 6(a) (see the caption for full details). The two spin-polarized modes obtained from $\boldsymbol{\alpha}_{\text {eff }}$ are interchanged upon band gap closing inside the ER (between points $Q$ and $R$ ), just like in the analogous tight-binding eigenfrequency analysis, which is presented in Fig. 6(b) for qualitative comparison. This shows that the features observed in k- $\omega$ maps in Figs. 4(a)-4(d), 5(b)-5(d), and $5(\mathrm{f})-5(\mathrm{~h})$ are indeed associated with ERs, and each EP that belongs to such ERs can be assigned a fractional topological charge according to Eq. (5) in the parameter space of in-plane momentum and resonant frequency detuning. In contrast, a Hermitian lattice with uniform gain does not show mode swapping upon following the same closed trajectory, as 

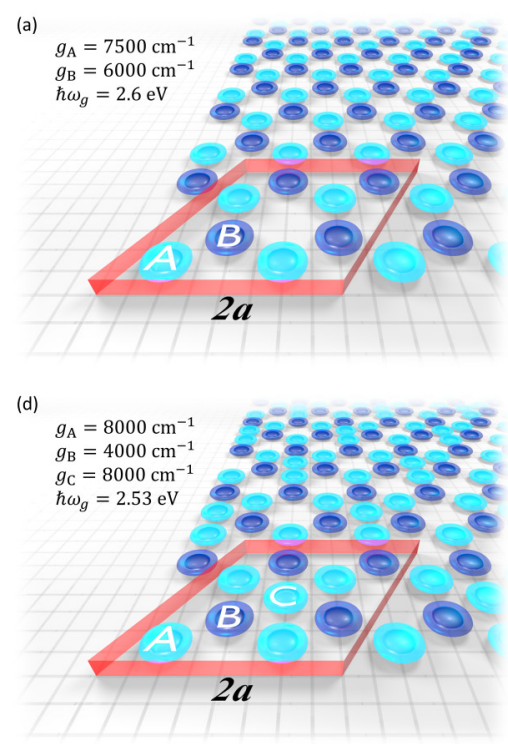
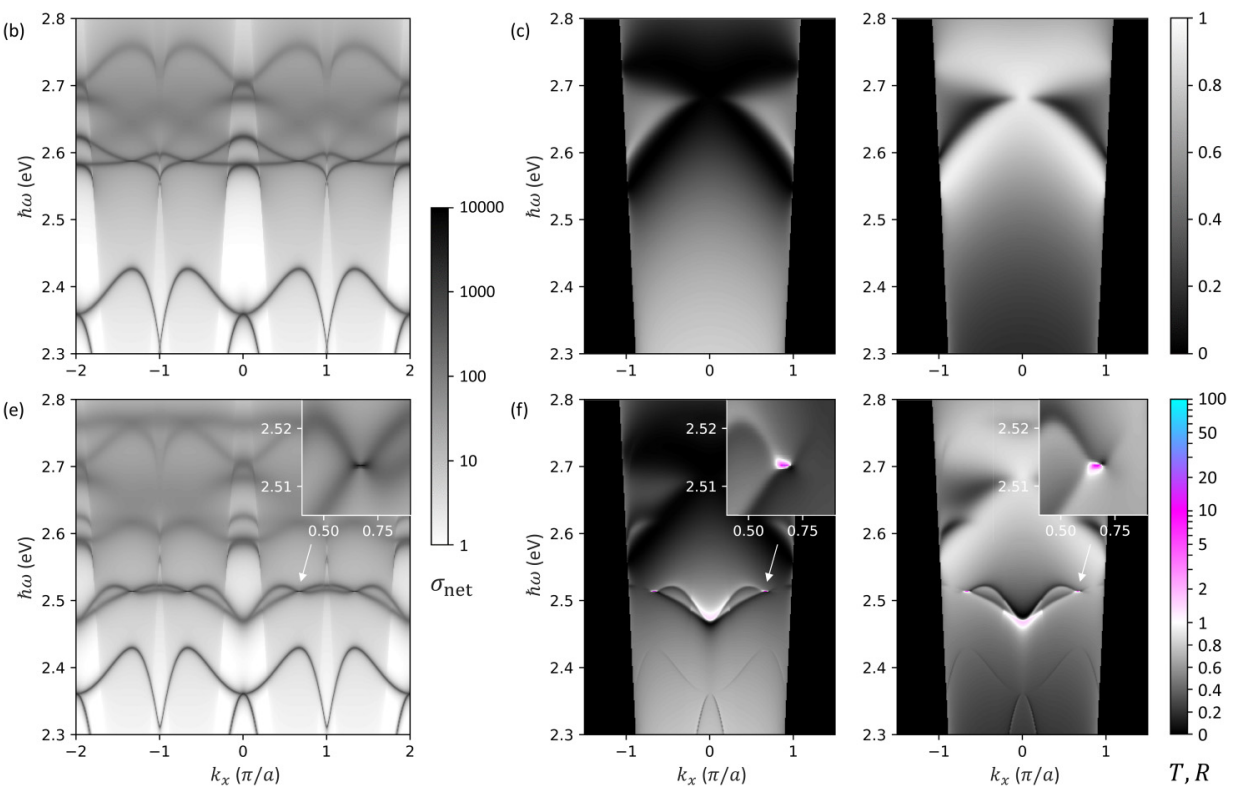

FIG. 7. Band folding in non-Hermitian honeycomb metasurfaces. (a) Scheme of the metasurface with a virtually extended unit cell. (b) Corresponding $\mathbf{k}_{\|}-\omega$ map of the net extinction, showing folded multiple copies of the original (unfolded) lattice modes visible in Fig. 4(a), but with only positive values of $\sigma_{\text {net }}$, as in Fig. 5(f). (c) Zeroth-order transmissivity and reflectivity under illumination by a plane wave linearly polarized at $45^{\circ}$, propagating from bottom $(-z)$ to top $(+z)$. The same scattering response would be obtained from calculations assuming the minimal unit cell, as in Fig. 1. (d) Modified honeycomb lattice with additional nanoparticles forming a new sublattice $C$. (e) Net extinction map showing new lattice resonances with flat bands associated with ERs (one of which is magnified in the inset). (f) These flat bands and the neighboring dispersive modes become visible in transmission and reflection, with remarkable amplification at the newly formed ERs (insets).

evident from Fig. 6(c), which is also in qualitative agreement with the results of eigendecomposition of the analogous tightbinding lattice Hamiltonian presented in Fig. 6(d).

The above examples demonstrate the suitability of the lattice Green's-function approach for non-Hermitian engineering of band topology in concrete physical systems, such as active plasmonic metasurfaces considered in this work. In addition, properly implemented calculations based on effective lattice polarizability and lattice Green's function are capable of providing quantitative predictions of measurable physical quantities such as scattering amplitudes, taking as an input concrete values of physical parameters. Examples of such implementation will be presented in the following section.

\section{CIRCULAR DICHROISM IN SCATTERING FROM EXCEPTIONAL RINGS}

In this section we will demonstrate the effect of $\mathcal{P} \mathcal{T}$ symmetry breaking on the far-field scattering response of an active plasmonic metasurface. In particular, we will show that the incoming and outgoing plane waves can be coupled to ERs by matching their in-plane momentum through band folding, achieved by appropriate modification of the lattice geometry, resulting in a differential scattering response to circularly polarized light at oblique incidence. Before presenting these results, we will briefly introduce the formulas for calculating the far-field scattering amplitudes, based on the effective lattice polarizability presented in Sec. III.

\section{A. Calculating transmission and reflection from the effective lattice polarizability}

In scattering of incident plane waves from periodic metasurfaces, the wave vectors $\mathbf{k}_{\tilde{m} \tilde{n}}$ of subsequent diffraction orders $\tilde{m}$ and $\tilde{n}$ can be expressed as

$$
\mathbf{k}_{\tilde{m} \tilde{n}}=k\left(\begin{array}{c}
\tilde{x} \\
\tilde{y} \\
\tilde{z}
\end{array}\right)=\left(\frac{\mathbf{k}_{\|}+\mathbf{K}_{\tilde{m} \tilde{n}}}{\sqrt{k^{2}-\left|\mathbf{k}_{\|}+\mathbf{K}_{\tilde{m} \tilde{n}}\right|^{2}}}\right),
$$

where $\mathbf{K}_{\tilde{m} \tilde{n}}=\tilde{m} \mathbf{b}_{1}+\tilde{n} \mathbf{b}_{2}$ are reciprocal lattice vectors $\left(\mathbf{a}_{i} \cdot \mathbf{b}_{j}=\delta_{i j}\right)$. For propagating diffraction orders, i.e., for $\left|\mathbf{k}_{\|}+\mathbf{K}_{\tilde{m} \tilde{n}}\right| \leqslant k$, these wave vectors can be converted to scattering angles $\theta$ and $\phi$,

$$
\mathbf{k}_{\tilde{m} \tilde{n}} \equiv \mathbf{k}(\theta, \phi)=k\left(\begin{array}{c}
\tilde{x} \\
\tilde{y} \\
\tilde{z}
\end{array}\right)=k\left(\begin{array}{c}
\sin \theta \cos \phi \\
\sin \theta \sin \phi \\
\cos \theta
\end{array}\right) .
$$

After calculating $\boldsymbol{\alpha}_{\text {eff }}\left(\omega, \mathbf{k}_{\|}\right)$using Eq. (15), the scattered fields associated with diffraction orders $\tilde{m}$ and $\tilde{n}$ can be obtained from

$$
\mathbf{E}_{\text {scat }}(\theta, \phi)= \pm \frac{2 \pi i k}{S \tilde{z}} \mathbf{M}(\theta, \phi) \sum_{l=1}^{N} \mathbf{p}_{l}\left(\omega, \mathbf{k}_{\|}\right) e^{-i \mathbf{k}(\theta, \phi) \cdot \mathbf{r}_{l}},
$$

where $S=\left|\mathbf{a}_{1} \times \mathbf{a}_{2}\right|$ is the unit cell area, $\mathbf{p}_{l}\left(\omega, \mathbf{k}_{\|}\right)$are the dipole moments calculated in Eq. (14), \pm is either + or depending on scattering direction (forward vs backward), and 
the far-field matrix $\mathbf{M}(\theta, \phi)$ is [75,77]

$$
\mathbf{M}(\theta, \phi)=\left(\begin{array}{ccc}
1-\tilde{x} \tilde{x} & -\tilde{x} \tilde{y} & -\tilde{x} \tilde{z} \\
-\tilde{y} \tilde{x} & 1-\tilde{y} \tilde{y} & -\tilde{y} \tilde{z} \\
-\tilde{z} \tilde{x} & -\tilde{z} \tilde{y} & 1-\tilde{z} \tilde{z}
\end{array}\right) .
$$

The transmissivity and the reflectivity are

$$
\begin{aligned}
T(\theta, \phi) & =-\frac{\operatorname{Re}\left[\mathbf{E}^{*}(\theta, \phi) \times \mathbf{H}(\theta, \phi)\right] \cdot \hat{\mathbf{z}}}{\operatorname{Re}\left[\mathbf{E}_{\mathrm{inc}}^{*}(\theta, \phi) \times \mathbf{H}_{\mathrm{inc}}(\theta, \phi)\right] \cdot \hat{\mathbf{z}}}, \\
R(\theta, \phi) & =\frac{\operatorname{Re}\left[\mathbf{E}_{\mathrm{scat}}^{*}(\pi-\theta, \phi) \times \mathbf{H}_{\mathrm{scat}}(\pi-\theta, \phi)\right] \cdot \hat{\mathbf{z}}}{\operatorname{Re}\left[\mathbf{E}_{\mathrm{inc}}^{*}(\theta, \phi) \times \mathbf{H}_{\mathrm{inc}}(\theta, \phi)\right] \cdot \hat{\mathbf{z}}},
\end{aligned}
$$

where

$$
\begin{aligned}
& \mathbf{E}(\theta, \phi)=\mathbf{E}_{\text {scat }}(\theta, \phi)+\mathbf{E}_{\text {inc }}(\theta, \phi), \\
& \mathbf{H}(\theta, \phi)=\mathbf{H}_{\text {scat }}(\theta, \phi)+\mathbf{H}_{\text {inc }}(\theta, \phi),
\end{aligned}
$$

with $\mathbf{E}_{\mathrm{inc}}$ and $\mathbf{H}_{\mathrm{inc}}$ the electric and magnetic fields of incident plane waves [Eq. (9)].

\section{B. $K$-point exceptional rings folded into the light cone}

In subdiffractive honeycomb metasurfaces, ERs are located outside the light cone, which makes them inaccessible from the far field. Increasing the lattice pitch is the simplest strategy that could bring them into the light cone by decreasing $\left|\mathbf{k}_{\|}\right|$of the $K$ points. Unfortunately, radiative damping of many overlapping diffraction orders would strongly distort the ERs. Here we implement another strategy, namely, band folding [91], which is illustrated in Fig. 7. In the first step, we artificially extend the unit cell, creating a supercell that covers four elementary cells and eight nanoparticles [Fig. 7(a)], giving rise to additional modes [Fig. 7(b)], which are just copies of the modes of original (unfolded) honeycomb metasurface and show no additional scattering signatures [Fig. 7(c)]. In the second step, we add one more nanoparticle inside each supercell, creating effectively a new sublattice $C$ [Fig. 7(d)] which preserves threefold rotational symmetry that protects the Dirac point degeneracies. Gain parameters are adjusted to $g_{C}=g_{A}=8000 \mathrm{~cm}^{-1}, g_{B}=4000 \mathrm{~cm}^{-1}$, and $\hbar \omega_{g}=2.53 \mathrm{eV}$. Sublattice $C$ couples with the other two sublattices, forming additional red-detuned ERs inside the light cone [Fig. 7(e)], with visible signatures in transmission and reflection [Fig. 7(f)]. Although net extinction remains positive, the metasurface shows a nonunitary scattering behavior $(T, R \gg 1)$ at the newly formed ERs (insets). To obtain significant effects in far-field observables, we found that the additional particles require polarizabilities as large as those of the particles forming the initial lattice.

\section{Nonunitary pseudochiral response at exceptional rings}

Figure 8(a) shows the scattering signatures of folded Dirac bands and ERs, simulated for various polarizations of incident light. The optical response is perfectly mirror symmetric with respect to $k_{x}=0$ under pure $s$ - and pure $p$-polarized illumination, but gets slightly asymmetric under linear polarization at $45^{\circ}$, evidencing coupling between in-plane Cartesian field components. This asymmetry is greatly augmented in the response to circular polarization and the pattern is mirror reversed upon switching handedness from right $(R)$ to left
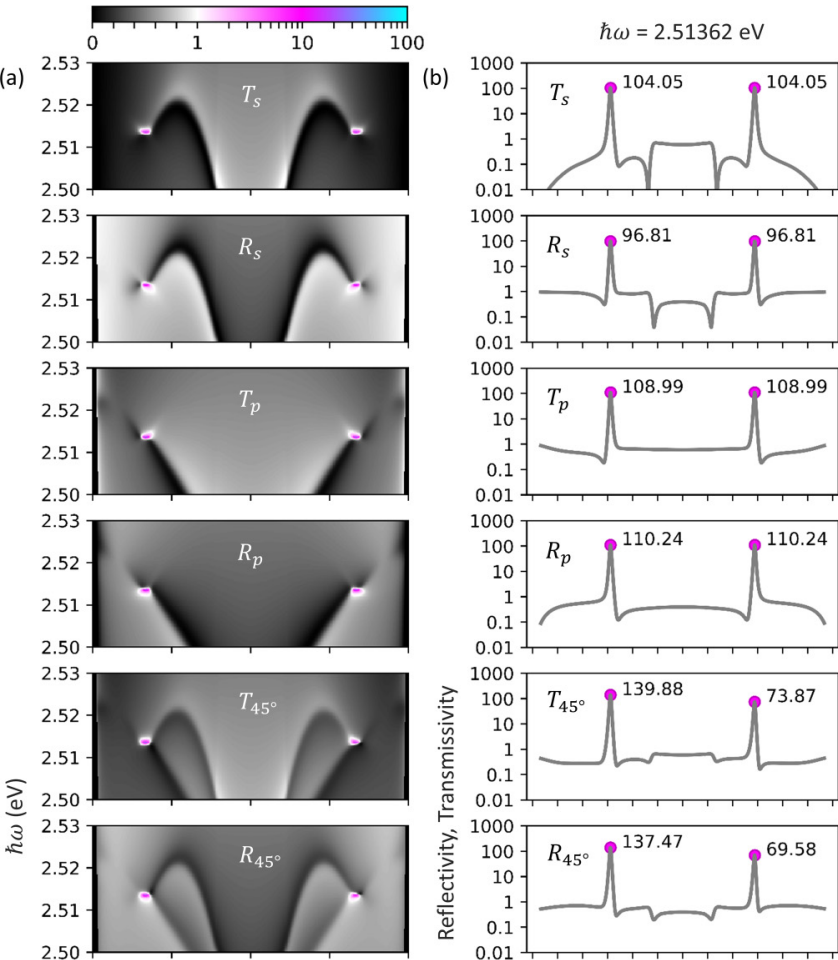

2.50

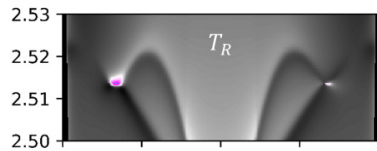

2.50

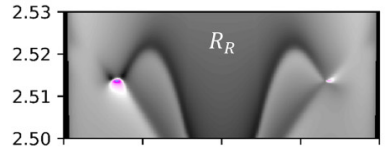

2.50

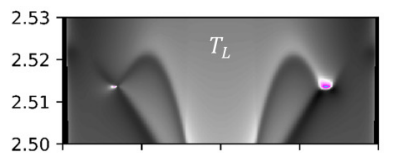

2.50
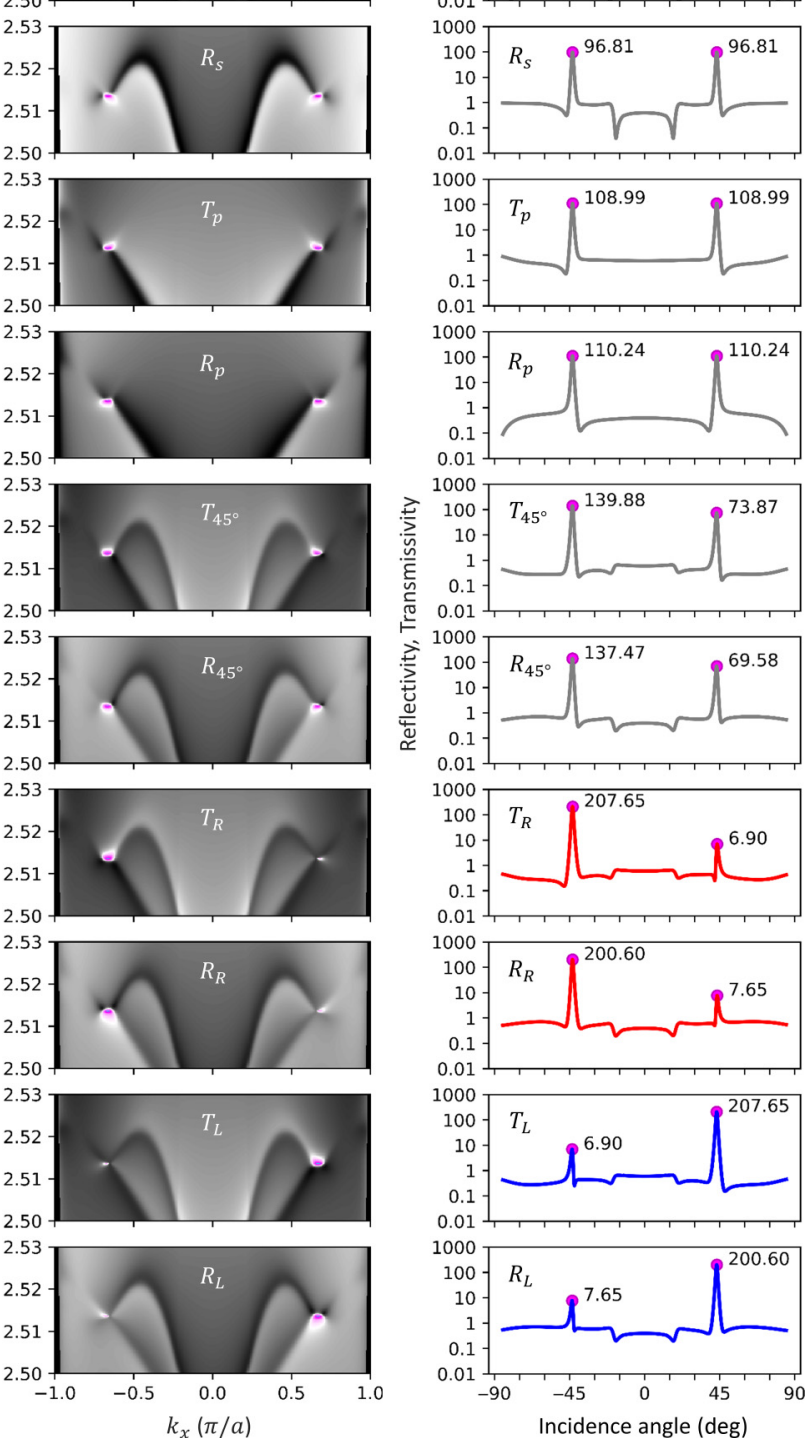

FIG. 8. Zeroth-order transmissivity $T$ and reflectivity $R$ of the metasurface shown in Fig. 7(d) calculated for various polarizations of incident light, i.e., linear $s\left(T_{s}\right.$ and $\left.R_{s}\right)$, linear $p\left(T_{p}\right.$ and $\left.R_{p}\right)$, linear at $45^{\circ}\left(T_{45^{\circ}}\right.$ and $\left.R_{45^{\circ}}\right)$, circular right-handed $\left(T_{R}\right.$ and $\left.R_{R}\right)$, and circular left-handed $\left(T_{L}\right.$ and $\left.R_{L}\right)$, (a) as a function of $k_{x}$ and $\omega$ and (b) as a function of incidence angle at a fixed photon energy $\hbar \omega=2.51362 \mathrm{eV}$. Peak values of amplified $T$ and $R$ are given in (b) next to the corresponding data points in angular dependence.

(L), which is schematically illustrated in Fig. 9(a) and presented more quantitatively in the fixed-frequency crosscuts in Fig. 8(b), with circular dichroism reaching $T_{R}-T_{L}= \pm 201$ in transmission and $R_{R}-R_{L}= \pm 193$ in reflection, where \pm depends on the sign of $k_{x}$ and both $T$ and $R$ are normalized by incident light intensity according to Eq. (27).

Such chiroptical effects at nonzero in-plane momentum, known as pseudochirality [92-95], have been extensively studied in passive systems [80,96,97]. As opposed to 
(a)
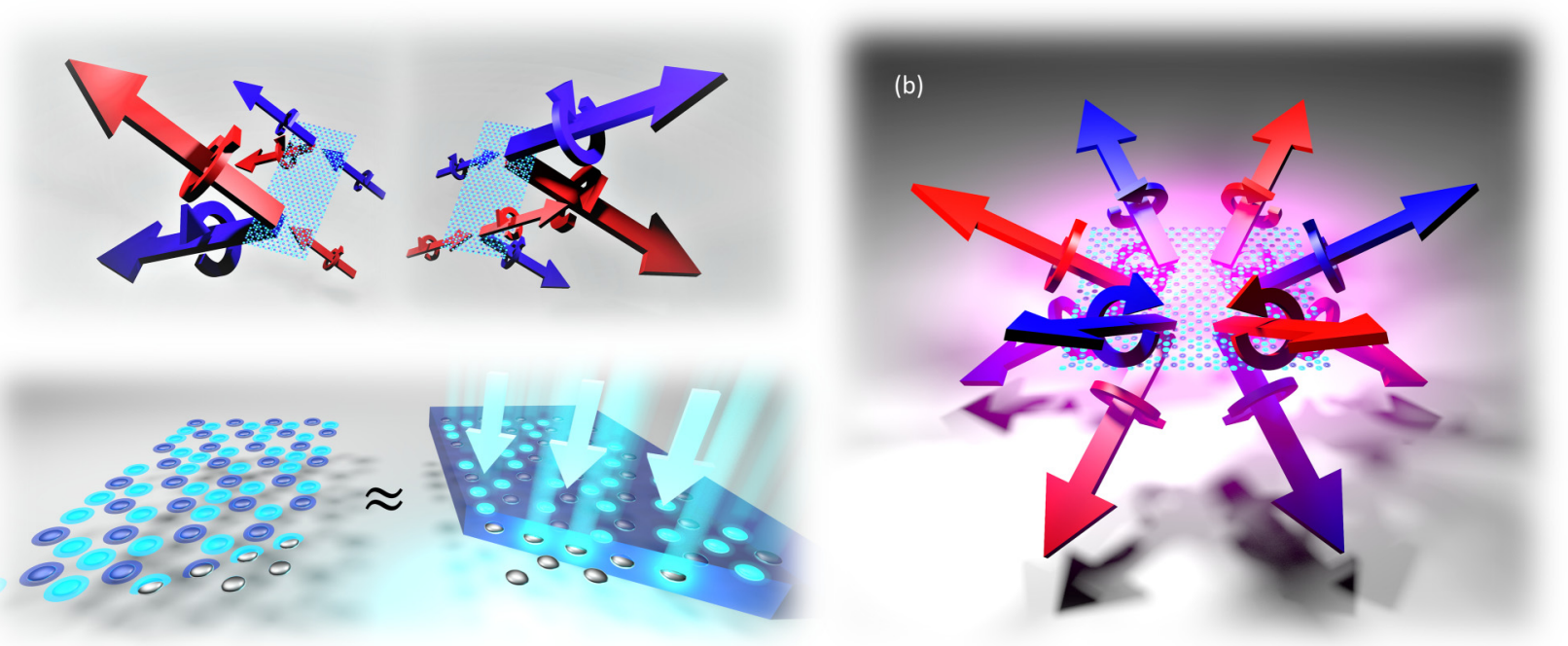

(c)

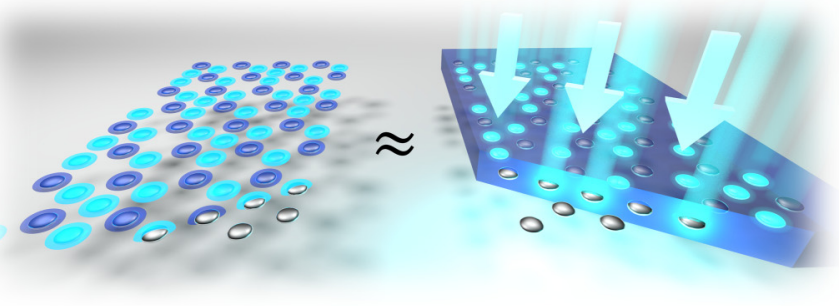

FIG. 9. (a) Illustration of the nonunitary pseudochiral circular dichroism at ERs below the lasing threshold. The incoming circularly polarized probe propagates from bottom $(-z)$ to top $(+z)$ at an oblique incidence angle and undergoes handedness- and direction-dependent amplification in transmission and reflection. (b) Extreme case of diverging circular dichroism at a scattering anomaly of the lasing threshold, equivalent to pseudochiral spin-polarized lasing at ERs. (c) Illustration of the approximation in which core-shell nanoparticle arrays serve as a model for traditional plasmon lattice lasers with metal nanoparticles embedded in a continuous gain medium layer under spatially varying optical pump fields.

passive systems, in our case the circular dichroism can easily exceed \pm 1 due to amplification and can in principle reach arbitrary magnitude when approaching the lasing threshold, at which scattering coefficients diverge [85,88,98], turning our system into a spin-polarized laser [Fig. 9(b)]. However, nonunitary circular dichroism is by itself not very astonishing, as it could be realized in ordinary pseudochiral systems upon introduction of gain. The most remarkable fact is that the pseudochiral response presented here is of nontrivial origin; it emerges from the gain-loss pattern and from the resulting ERs capable of interacting with incident and outgoing plane waves. A nonunitary pseudochiral response could be accessed experimentally in polarization-resolved pump-probe transient scatterometry, which could serve as an elegant method for investigation of gain-induced $\mathcal{P} \mathcal{T}$-symmetry breaking. In the future, they could be used for realization of dynamically controlled polarization-converting metasurfaces.

\section{OUTLOOK AND SUMMARY}

\section{A. Feasibility of experimental realization}

Experimental realization of core-shell nanoparticle arrays presented in Figs. 1, 7(a), and 7(d) is technologically challenging. We have considered this particular design as an approximation of typical metasurface lasers made of plasmonic nanoparticle arrays embedded in continuous gain medium films illustrated in Fig. 9(c). Such geometries are easily achievable by well-established lithography and spincoating techniques. Moreover, they are known to reach lasing thresholds under moderate pump intensities and with gain coefficients on the order of a few tens of $\mathrm{cm}^{-1}$, despite the presence of lossy plasmonic scatterers. It is possible because efficient amplification of multiply scattered modes occurs upon propagation across the embedding gain medium.
In contrast, amplification upon scattering from a nanoparticle covered by a gain medium shell is highly inefficient, as it requires one to compensate for plasmonic losses in the nanoparticle's core. This is why in our calculations we assumed gain coefficients as high as a few thousand $\mathrm{cm}^{-1}$. Inspired by numerous successful demonstrations of plasmon lattice lasers [50,55-57,91], we believe that the presence of a continuous embedding gain medium would be a feasible route towards experimental realization of non-Hermitian pseudochiral phenomena discussed in this work, on the proviso that one can implement locally varying gain through, for instance, a spatially varying optical pump field [Fig. 9(c)].

The proviso of spatially varying gain and loss is experimentally not easily met due to the need for subwavelength length scales in the gain-loss patterns. Here we propose an elegant way to achieve that, hinging on the physics of Dirac points. Oblique illumination of a honeycomb lattice by circularly polarized light at a Dirac point enables selective excitation of one of the two sublattices (either $A$ or $B$ ). In such a case, one may expect that the optical gain will be localized in places where the gain medium is pumped most effectively, i.e., in the vicinity of plasmonic nanoparticles that belong to the sublattice that is more effectively excited by the pump beam. Obviously, efficient excitation requires that such Dirac points lie within the light cone, which we have already achieved in the design presented in Fig. 7(d). Let us consider plasmonic metasurface of similar geometry, but without the gain medium shell, as illustrated in Fig. 10(a). Since the pump does not experience gain, all modes are strongly blurred in frequency; however, their symmetries and topologies are robust against spectral broadening. We calculate the degree of preferential excitation of sublattice $A$ against sublattice $B$ under circularly polarized illumination and we present the results as a k- $\omega$ map in Fig. 10(b). Interestingly, the difference 
(a)
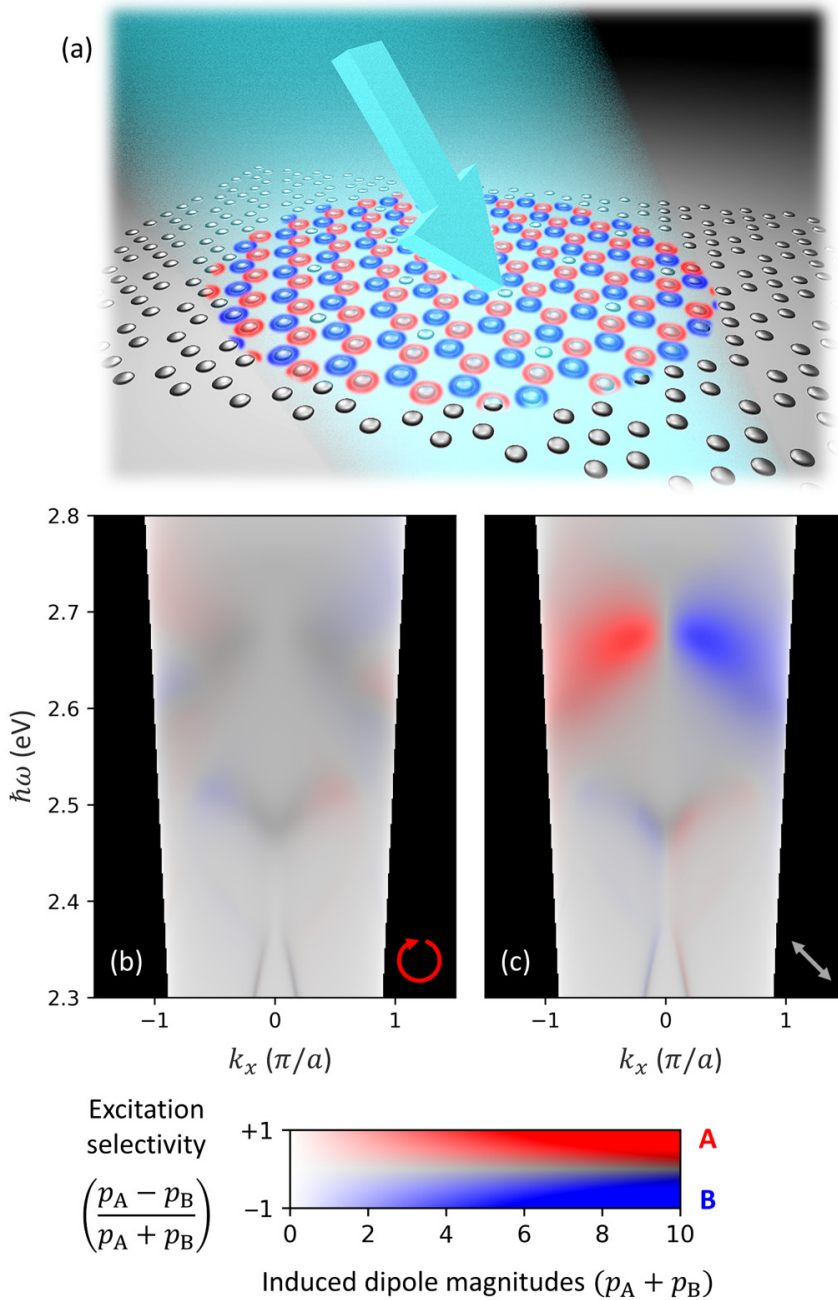

FIG. 10. (a) Illustration of optical pumping scheme to achieve selective excitation of sublattices by polarized illumination of the metasurface embedded in a homogeneous gain medium, leading to a gain contrast between sublattices $A$ and $B$. Red and blue "clouds" around nanoparticles indicate efficient excitation of either sublattice $A$ or $B$, respectively, which could create effective gain shells providing amplification of a lower-frequency probe beam. (b) Preferential excitation of sublattice $A$ versus sublattice $B$, quantified by the average magnitude of dipole moments induced in these sublattices by right-handed circularly polarized light propagating from top $(+z)$ to bottom $(-z)$. (c) Same as (b) but for linearly polarized excitation at $-45^{\circ}$. Surprisingly, the contrast is much greater compared to circularly polarized pumping and in principle should allow for highly selective excitation of one sublattice, creating a gain-loss contrast effective in the frequency range of desired ER formation.

between average magnitudes of the dipole moments induced in sublattices $A$ and $B$ is only moderate under circularly polarized illumination at the folded Dirac points, but becomes very large under illumination that is linearly polarized at $-45^{\circ}$ (equivalent to $+45^{\circ}$ for a beam propagating from bottom to top), which is shown in Fig. 10(c). The highest contrast is not associated with Dirac points designated to become ERs, as it is observed at frequencies larger than the frequencies at which ERs are expected to emerge. As a result, this scheme is highly promising in terms of efficient pumping of a gain medium to create gain-loss patterns resulting in ERs discussed in this work. Apart from the above idea, there are probably many other routes to pseudochiral ER scattering physics, possibly including gain-free designs [14,99], which certainly deserve further investigation.

\section{B. Spin-polarized exceptional ring laser}

Our theoretical predictions are accurate up to the lasing threshold, where unstable solutions with negative extinction start to emerge. Nevertheless, some results obtained in this regime can be used to qualitatively predict the behavior of a lasing system. Slightly above the lasing threshold [Figs. 5(c), $5(\mathrm{~g})$, and $5(\mathrm{k})]$, the gain-induced changes in polarizability inside ERs would contribute to mode competition, leading to protection of net gain by the gain-loss contrast. Adding loss to one sublattice would paradoxically improve the performance of such a laser $[100,101]$, even without adding an equivalent amount of gain to the other sublattice. Such exceptional ring lasers could serve as integrated coherent light sources, a nonHermitian counterpart of spin-momentum-locked topological lasers [63-65]. As opposed to systems with pairs of isolated EPs in momentum space, connected by flat bands in a form of Fermi arcs [40], flat bands inside ERs span finite areas across $k_{x}$ and $k_{y}$, promising a much larger density of optical states, which could serve as an alternative to band edge distributed feedback lasers. The folded version of ER lasing [the design in Fig. 7(d)] would provide coherent spin-polarized emission at off-normal angles [Fig. 9(b)], extending the family of Dirac-point surface-emitting lasers [68-70]. Exceptional ring lasers could be implemented also in nonplasmonic lattices [102] with potentially lower gain coefficients. Rigorous performance analysis [84,89] of various kinds of ER-based lasing metasurfaces, including the possibility of single-mode lasing $[69,103]$ and the effect of gain saturation and dynamics [64,86,90,104-106], will be an exciting aspect of their further exploration.

\section{Conclusions}

In conclusion, we have shown that breaking $\mathcal{P} \mathcal{T}$ symmetry in active plasmonic metasurfaces can lead to a pseudochiral optical response accompanied by strong amplification in reflection and transmission, leading to nonunitary circular dichroism. This has been demonstrated by calculating the scattering observables of a modified honeycomb metasurface, in which band folding gives rise to ERs inside the light cone, coupling their spin-polarized flat bands with farfield radiation. By comparing results obtained by the lattice Green's-function method with those predicted by a simple toy model based on a vectorial tight-binding Hamiltonian, we show that the pseudospins of flat bands inside ERs are topologically linked to pseudospins of valleys in gapped Hermitian honeycomb lattices. Moreover, we show that net ellipticity and pseudochiral optical response of these bands result from the effect of gain and loss on their spectral linewidth and polarizability magnitude. Such effects are not accounted for in simple tight-binding Hamiltonian models. The above results signify the usefulness of rigorous Green's-function methods, such as the one used in our work, for predicting the 
scattering response of active non-Hermitian metasurfaces. We believe that our findings are applicable in designing active polarization-converting and light-emitting devices, including integrated or surface-emitting spin-polarized ER lasers with robust operation protected by the gain-loss contrast.

\section{ACKNOWLEDGMENTS}

This work was performed at the research institute AMOLF, as part of the research program Hybrid Nanophotonic Architectures for Ultrafast Quantum Optics (Project No. 680.47.621) and of the research program Nanophotonics for Solid-State Lighting (Project FOM-i33/680.93.33), which both are partly financed by the Dutch Research Council (NWO).

\section{APPENDIX A: LATTICE SUM CALCULATIONS}

The lattice sums can be decomposed into exponentially converging reciprocal-space and real-space summations $[107,108]$, whose scalar forms are

$$
\begin{aligned}
\sum_{m, n} \frac{e^{i k \rho_{m n}}}{\rho_{m n}} e^{i \mathbf{k} \cdot \mathbf{R}_{m n}}= & \frac{\pi}{S} \sum_{\tilde{m}, \tilde{n}} e^{i k(\tilde{x}, \tilde{y})^{T} \cdot \mathbf{r}_{\|}} f_{\tilde{m} \tilde{n}}^{(1)}(z) \\
& +\sum_{m, n} e^{i \mathbf{k}_{\|} \cdot \mathbf{R}_{m n}} f^{(2)}\left(\rho_{m n}\right),
\end{aligned}
$$

where $\rho_{m n}=\left|\mathbf{r}-\mathbf{R}_{m n}\right|, \mathbf{r}=\left(\mathbf{r}_{\|}, z\right)^{T}$, and

$$
\begin{aligned}
& f_{\tilde{m} \tilde{n}}^{(1)}(z)=\frac{F(k \tilde{z},|z|)+F(k \tilde{z},-|z|)}{k \tilde{z}}, \\
& f^{(2)}\left(\rho_{m n}\right)=\frac{F^{\prime}\left(i k, \rho_{m n}\right)+F^{\prime}\left(-i k, \rho_{m n}\right)}{2 \rho_{m n}}, \\
& F(p, q)=e^{i p q} \operatorname{erfc}\left(\frac{p}{2 \eta}+q \eta\right), \\
& F^{\prime}(p, q)=e^{p q} \operatorname{erfc}\left(\frac{p}{2 \eta}+q \eta\right) .
\end{aligned}
$$

Good convergence is achieved by setting $\eta=\sqrt{\pi} / a$, where $a$ is the lattice pitch. In general, summations in Eq. (A1) should run until $|m|,|n|,|\tilde{m}|,|\tilde{n}| \leqslant 5$; however, we found that 4 is actually sufficient in all cases studied in this article. Evaluation of Eq. (12) requires computing the complete sum that includes the lattice origin and then subtracting the zeroth term, which is calculated as the average of the $\mathbf{G}^{0}$ obtained from Eq. (7) by setting $\mathbf{r}^{\prime}=\mathbf{R}_{00}$ and at two symmetric offsets $\mathbf{r}=\mathbf{r}_{\mathrm{off}}$ to avoid singularity. Dyadic forms of the terms in Eq. (A1) are

$$
\begin{aligned}
\left(\mathbb{I} k^{2}+\right. & \nabla \otimes \nabla) e^{i k(\tilde{x}, \tilde{y})^{T} \cdot \mathbf{r}_{\|}} f_{\tilde{m} \tilde{n}}^{(1)}(z) \\
& =e^{i k(\tilde{x}, \tilde{y})^{T} \cdot \mathbf{r}_{\|}}\left[\left(\begin{array}{ccc}
1-\tilde{x}^{2} & -\tilde{x} \tilde{y} & 0 \\
-\tilde{x} \tilde{y} & 1-\tilde{y}^{2} & 0 \\
0 & 0 & 1
\end{array}\right) k^{2}\right. \\
& \left.+\left(\begin{array}{ccc}
0 & 0 & i \tilde{x} \\
0 & 0 & i \tilde{y} \\
i \tilde{x} & i \tilde{y} & 0
\end{array}\right) k \frac{d}{d z}+\left(\begin{array}{ccc}
0 & 0 & 0 \\
0 & 0 & 0 \\
0 & 0 & 1
\end{array}\right) \frac{d^{2}}{d z^{2}}\right] f_{\tilde{m} \tilde{n}}^{(1)}(z), \\
\left(\mathbb{I} k^{2}+\right. & \nabla \otimes \nabla) f^{(2)}\left[\rho_{m n}(r)\right]=\left[\mathbb{I}\left(k^{2}+\frac{1}{r} \frac{d}{d r}\right)\right. \\
& \left.+\left(\begin{array}{lll}
x^{2} & x y & x z \\
x y & y^{2} & y z \\
x z & y z & z^{2}
\end{array}\right) \frac{1}{r} \frac{d}{d r} \frac{1}{r} \frac{d}{d r}\right] f^{(2)}\left[\rho_{m n}(r)\right] .
\end{aligned}
$$

\section{APPENDIX B: POLARIZABILITY OF A CORE-SHELL ELLIPSOID}

Static electric point dipole polarizability of a core-shell ellipsoid is [81]

$$
\boldsymbol{\alpha}_{\text {stat }}=\frac{V}{4 \pi}\left(\begin{array}{ccc}
A_{x} & 0 & 0 \\
0 & A_{y} & 0 \\
0 & 0 & A_{z}
\end{array}\right)
$$

where $A_{j}$ are defined for each symmetry axis $j=x, y, z$,

$$
A_{j}=\frac{B_{j}^{-1}-\beta C_{j}^{-1}}{\left(B_{j} D_{j}\right)^{-1}+\beta L_{2, j}\left(1-L_{2, j}\right)},
$$

where $\beta$ is the volume fraction of the core and

$$
\begin{gathered}
B_{j}=\frac{\varepsilon_{3}-\varepsilon_{2}}{L_{3, j}\left(\varepsilon_{3}-\varepsilon_{2}\right)+\varepsilon_{2}}, \\
C_{j}=\frac{\varepsilon_{1}-\varepsilon_{2}}{L_{2, j}\left(\varepsilon_{1}-\varepsilon_{2}\right)+\varepsilon_{2}}, \\
D_{j}=\frac{\varepsilon_{2}-\varepsilon_{1}}{L_{2, j}\left(\varepsilon_{2}-\varepsilon_{1}\right)+\varepsilon_{1}},
\end{gathered}
$$

with shell permittivity $\varepsilon_{2}$ and core permittivity $\varepsilon_{3}$, embedded in a medium of permittivity $\varepsilon_{1}$, and with shell and core shape factors $L_{2, j}$ and $L_{3, j}$, respectively, expressed in terms of the subsequent radii $r_{j}$ of the ellipsoid [87]

$$
L_{j}=\frac{r_{x} r_{y} r_{z}}{2} \int_{0}^{\infty} \frac{d s}{\left(s+r_{j}^{2}\right) \sqrt{\left(s+r_{x}^{2}\right)\left(s+r_{y}^{2}\right)\left(s+r_{z}^{2}\right)}}
$$

[1] N. Yu, P. Genevet, M. A. Kats, F. Aieta, J.-P. Tetienne, F. Capasso, and Z. Gaburro, Light propagation with phase discontinuities: generalized laws of reflection and refraction, Science 334, 333 (2011).

[2] A. Silva, F. Monticone, G. Castaldi, V. Galdi, A. Alù, and N. Engheta, Performing mathematical operations with metamaterials, Science 343, 160 (2014).
[3] H.-T. Chen, A. J. Taylor, and N. Yu, A review of metasurfaces: Physics and applications, Rep. Prog. Phys. 79, 076401 (2016).

[4] S. M. Kamali, E. Arbabi, A. Arbabi, and A. Faraon, A review of dielectric optical metasurfaces for wavefront control, Nanophotonics 7, 1041 (2018). 
[5] M. Botey, R. Herrero, and K. Staliunas, Light in materials with periodic gain-loss modulation on a wavelength scale, Phys. Rev. A 82, 013828 (2010).

[6] R. Fleury, D. L. Sounas, and A. Alù, Negative Refraction and Planar Focusing Based on Parity-Time Symmetric Metasurfaces, Phys. Rev. Lett. 113, 023903 (2014).

[7] A. Cerjan, A. Raman, and S. Fan, Exceptional Contours and Band Structure Design in Parity-Time Symmetric Photonic Crystals, Phys. Rev. Lett. 116, 203902 (2016).

[8] C. W. Ling, K. H. Choi, T. C. Mok, Z.-Q. Zhang, and K. H. Fung, Anomalous light scattering by topological PTsymmetric particle arrays, Sci. Rep. 6, 38049 (2016).

[9] F. Monticone, C. A. Valagiannopoulos, and A. Alù, ParityTime Symmetric Nonlocal Metasurfaces: All-Angle Negative Refraction and Volumetric Imaging, Phys. Rev. X 6, 041018 (2016).

[10] C. Hahn, E. K. Keshmarzi, S. H. Song, C. H. Oh, R. N. Tait, and P. Berini, Unidirectional Bragg gratings using parity-time symmetry breaking in plasmonic systems, IEEE J. Sel. Top. Quantum Electron. 22, 48 (2016).

[11] M. Sakhdari, M. Farhat, and P.-Y. Chen, PT-symmetric metasurfaces: Wave manipulation and sensing using singular points, New J. Phys. 19, 065002 (2017).

[12] H. M. Leung, W. Gao, R. Zhang, Q. Zhao, X. Wang, C. T. Chan, J. Li, and W. Y. Tam, Exceptional point-based plasmonic metasurfaces for vortex beam generation, Opt. Express 28, 503 (2020).

[13] Y. J. Zhang, P. Li, V. Galdi, M. S. Tong, and A. Alù, Manipulating the scattering pattern with non-Hermitian particle arrays, Opt. Express 28, 19492 (2020).

[14] B. Zhen, C. W. Hsu, Y. Igarashi, L. Lu, I. Kaminer, A. Pick, S.-L. Chua, J. D. Joannopoulos, and M. Soljačić, Spawning rings of exceptional points out of Dirac cones, Nature (London) 525, 354 (2015).

[15] L. Feng, R. El-Ganainy, and L. Ge, Non-Hermitian photonics based on parity-time symmetry, Nat. Photonics 11, 752 (2017).

[16] M.-A. Miri and A. Alù, Exceptional points in optics and photonics, Science 363, eaar7709 (2019).

[17] Ş. K. Özdemir, S. Rotter, F. Nori, and L. Yang, Parity-time symmetry and exceptional points in photonics, Nat. Mater. 18, 783 (2019).

[18] L. Feng, Y.-L. Xu, W. S. Fegadolli, M.-H. Lu, J. E. B. Oliveira, V. R. Almeida, Y.-F. Chen, and A. Scherer, Experimental demonstration of a unidirectional reflectionless parity-time metamaterial at optical frequencies, Nat. Mater. 12, 108 (2013).

[19] S. Dong, G. Hu, Q. Wang, Y. Jia, Q. Zhang, G. Cao, J. Wang, S. Chen, D. Fan, W. Jiang et al., Loss-assisted metasurface at an exceptional point, ACS Photon. 7, 3321 (2020).

[20] B. Peng, Ş. K. Özdemir, F. Lei, F. Monifi, M. Gianfreda, G. L. Long, S. Fan, F. Nori, C. M. Bender, and L. Yang, Paritytime-symmetric whispering-gallery microcavities, Nat. Phys. 10, 394 (2014).

[21] Z. Gao, S. T. M. Fryslie, B. J. Thompson, P. S. Carney, and K. D. Choquette, Parity-time symmetry in coherently coupled vertical cavity laser arrays, Optica 4, 323 (2017).

[22] B. Peng, Ş. K. Özdemir, M. Liertzer, W. Chen, J. Kramer, H. Yllmaz, J. Wiersig, S. Rotter, and L. Yang, Chiral modes and directional lasing at exceptional points, Proc. Natl. Acad. Sci. USA 113, 6845 (2016).
[23] P. Miao, Z. Zhang, J. Sun, W. Walasik, S. Longhi, N. M. Litchinitser, and L. Feng, Orbital angular momentum microlaser, Science 353, 464 (2016).

[24] M. Lawrence, N. Xu, X. Zhang, L. Cong, J. Han, W. Zhang, and S. Zhang, Manifestation of $P T$ Symmetry Breaking in Polarization Space with Terahertz Metasurfaces, Phys. Rev. Lett. 113, 093901 (2014).

[25] J. Doppler, A. A. Mailybaev, J. Böhm, U. Kuhl, A. Girschik, F. Libisch, T. J. Milburn, P. Rabl, N. Moiseyev, and S. Rotter, Dynamically encircling an exceptional point for asymmetric mode switching, Nature (London) 537, 76 (2016).

[26] A. U. Hassan, B. Zhen, M. Soljačić, M. Khajavikhan, and D. N. Christodoulides, Dynamically Encircling Exceptional Points: Exact Evolution and Polarization State Conversion, Phys. Rev. Lett. 118, 093002 (2017).

[27] P. Ginzburg, F. J. Rodríguez-Fortuño, A. Martínez, and A. V. Zayats, Analogue of the quantum Hanle effect and polarization conversion in non-Hermitian plasmonic metamaterials, Nano Lett. 12, 6309 (2012).

[28] M. Kang and Y. D. Chong, Coherent optical control of polarization with a critical metasurface, Phys. Rev. A 92, 043826 (2015).

[29] A. Cerjan and S. Fan, Achieving Arbitrary Control Over Pairs of Polarization States using Complex Birefringent Metamaterials, Phys. Rev. Lett. 118, 253902 (2017).

[30] B. Baum, M. Lawrence, D. Barton, III, J. Dionne, and H. Alaeian, Active polarization control with a parity-timesymmetric plasmonic resonator, Phys. Rev. B 98, 165418 (2018)

[31] J. Li, J. Fu, Q. Liao, and S. Ke, Exceptional points in chiral metasurface based on graphene strip arrays, J. Opt. Soc. Am. B 36, 2492 (2019).

[32] S. H. Park, S.-G. Lee, S. Baek, T. Ha, S. Lee, B. Min, S. Zhang, M. Lawrence, and T.-T. Kim, Observation of an exceptional point in a non-Hermitian metasurface, Nanophotonics $\mathbf{9}, 1031$ (2020).

[33] C. Dembowski, H.-D. Gräf, H. L. Harney, A. Heine, W. D. Heiss, H. Rehfeld, and A. Richter, Experimental Observation of the Topological Structure of Exceptional Points, Phys. Rev. Lett. 86, 787 (2001).

[34] M. Berry, Optical polarization evolution near a non-Hermitian degeneracy, J. Opt. 13, 115701 (2011).

[35] M. Kang, J. Chen, and Y. D. Chong, Chiral exceptional points in metasurfaces, Phys. Rev. A 94, 033834 (2016).

[36] S. Yu, H. S. Park, X. Piao, B. Min, and N. Park, Lowdimensional optical chirality in complex potentials, Optica 3, 1025 (2016).

[37] S. Yu, X. Piao, and N. Park, Chirality in non-Hermitian photonics, Curr. Opt. Photon. 3, 275 (2019).

[38] T. Wu, W. Zhang, H. Zhang, S. Hou, G. Chen, R. Liu, C. Lu, J. Li, R. Wang, P. Duan et al., Vector Exceptional Points with Strong Superchiral Fields, Phys. Rev. Lett. 124, 083901 (2020).

[39] H. Shen, B. Zhen, and L. Fu, Topological Band Theory for Non-Hermitian Hamiltonians, Phys. Rev. Lett. 120, 146402 (2018).

[40] H. Zhou, C. Peng, Y. Yoon, C. W. Hsu, K. A. Nelson, L. Fu, J. D. Joannopoulos, M. Soljačić, and B. Zhen, Observation of bulk Fermi arc and polarization half charge from paired exceptional points, Science 359, 1009 (2018). 
[41] A. Szameit, M. C. Rechtsman, O. Bahat-Treidel, and M. Segev, $\mathscr{P} \mathscr{T}$-symmetry in honeycomb photonic lattices, Phys. Rev. A 84, 021806(R) (2011).

[42] C. W. Curtis and M. J. Ablowitz, On the existence of real spectra in $\mathcal{P} \mathcal{T}$-symmetric honeycomb optical lattices, J. Phys. A: Math. Theor. 47, 225205 (2014).

[43] Z. Oztas and C. Yuce, Spontaneously broken particle-hole symmetry in photonic graphene with gain and loss, Phys. Rev. A 98, 042104 (2018).

[44] M. Kremer, T. Biesenthal, L. J. Maczewsky, M. Heinrich, R. Thomale, and A. Szameit, Demonstration of a twodimensional $\mathcal{P} \mathcal{T}$-symmetric crystal, Nat. Commun. 10, 435 (2019).

[45] A. Cerjan, S. Huang, M. Wang, K. P. Chen, Y. Chong, and M. C. Rechtsman, Experimental realization of a Weyl exceptional ring, Nat. Photonics 13, 623 (2019).

[46] M. Turduev, M. Botey, I. Giden, R. Herrero, H. Kurt, E. Özbay, and K. Staliunas, Two-dimensional complex paritytime-symmetric photonic structures, Phys. Rev. A 91, 023825 (2015).

[47] M. Botey, R. Herrero, M. Turduev, I. Giden, H. Kurt, and K. Staliunas, Chiral modes in 2D PT-symmetric nanostructures, in Nonlinear Dynamics: Materials, Theory and Experiments, edited by M. Tlidi and M. G. Clerc (Springer, Berlin, 2016), pp. $125-138$.

[48] N. S. Nye, A. El Halawany, A. Bakry, M. A. N. Razvi, A. Alshahrie, M. Khajavikhan, and D. N. Christodoulides, Passive PT-symmetric metasurfaces with directional field scattering characteristics, IEEE J. Sel. Top. Quantum Electron. 22, 5000608 (2016).

[49] A. El Halawany, Optical parity time metasurface structures, $\mathrm{Ph} . \mathrm{D}$. thesis, University of Central Florida, 2016, available at https://stars.library.ucf.edu/etd/5303/.

[50] R. Guo, M. Nečada, T. K. Hakala, A. I. Väkeväinen, and P. Törmä, Lasing at $K$ Points of a Honeycomb Plasmonic Lattice, Phys. Rev. Lett. 122, 013901 (2019).

[51] R. Li, M. R. Bourgeois, C. Cherqui, J. Guan, D. Wang, J. Hu, R. D. Schaller, G. C. Schatz, and T. W. Odom, Hierarchical hybridization in plasmonic honeycomb lattices, Nano Lett. 19, 6435 (2019).

[52] R. Kolkowski and A. F. Koenderink, Lattice resonances in optical metasurfaces with gain and loss, Proc. IEEE 108, 795 (2019).

[53] H. Wang, B. Xie, S. K. Gupta, X. Zhu, L. Liu, X. Liu, M. Lu, and Y. Chen, Exceptional concentric rings in a non-Hermitian bilayer photonic system, Phys. Rev. B 100, 165134 (2019).

[54] N. S. Nye, A. E. Halawany, C. Markos, M. Khajavikhan, and D. N. Christodoulides, Flexible $\mathscr{P} \mathscr{T}$-Symmetric Optical Metasurfaces, Phys. Rev. Appl. 13, 064005 (2020).

[55] A. H. Schokker and A. F. Koenderink, Lasing at the band edges of plasmonic lattices, Phys. Rev. B 90, 155452 (2014).

[56] T. K. Hakala, H. T. Rekola, A. I. Väkeväinen, J.-P. Martikainen, M. Nečada, A. J. Moilanen, and P. Törmä, Lasing in dark and bright modes of a finite-sized plasmonic lattice, Nat. Commun. 8, 13687 (2017).

[57] D. Wang, W. Wang, M. P. Knudson, G. C. Schatz, and T. W. Odom, Structural engineering in plasmon nanolasers, Chem. Rev. 118, 2865 (2017).

[58] F. J. García de Abajo, Colloquium: Light scattering by particle and hole arrays, Rev. Mod. Phys. 79, 1267 (2007).
[59] T. Ma and G. Shvets, All-Si valley-Hall photonic topological insulator, New J. Phys. 18, 025012 (2016).

[60] J.-W. Dong, X.-D. Chen, H. Zhu, Y. Wang, and X. Zhang, Valley photonic crystals for control of spin and topology, Nat. Mater. 16, 298 (2017).

[61] M. I. Shalaev, W. Walasik, A. Tsukernik, Y. Xu, and N. M. Litchinitser, Robust topologically protected transport in photonic crystals at telecommunication wavelengths, Nat. Nanotechnol. 14, 31 (2019).

[62] M. Proctor, P. A. Huidobro, S. A. Maier, R. V. Craster, and M. P. Makwana, Manipulating topological valley modes in plasmonic metasurfaces, Nanophotonics 9, 657 (2020).

[63] Y. Zeng, U. Chattopadhyay, B. Zhu, B. Qiang, J. Li, Y. Jin, L. Li, A. G. Davies, E. H. Linfield, B. Zhang et al., Electrically pumped topological laser with valley edge modes, Nature (London) 578, 246 (2020).

[64] J.-S. Wu, V. Apalkov, and M. I. Stockman, Topological Spaser, Phys. Rev. Lett. 124, 017701 (2020).

[65] Z.-Q. Yang, Z.-K. Shao, H.-Z. Chen, X.-R. Mao, and R.M. Ma, Spin-Momentum-Locked Edge Mode for Topological Vortex Lasing, Phys. Rev. Lett. 125, 013903 (2020).

[66] W. H. Weber and G. W. Ford, Propagation of optical excitations by dipolar interactions in metal nanoparticle chains, Phys. Rev. B 70, 125429 (2004).

[67] A. F. Koenderink and A. Polman, Complex response and polariton-like dispersion splitting in periodic metal nanoparticle chains, Phys. Rev. B 74, 033402 (2006).

[68] J. Bravo-Abad, J. D. Joannopoulos, and M. Soljačić, Enabling single-mode behavior over large areas with photonic Dirac cones, Proc. Natl. Acad. Sci. USA 109, 9761 (2012).

[69] S.-L. Chua, L. Lu, J. Bravo-Abad, J. D. Joannopoulos, and M. Soljačić, Larger-area single-mode photonic crystal surfaceemitting lasers enabled by an accidental Dirac point, Opt. Lett. 39, 2072 (2014).

[70] S. L. Chua, L. Lu, and M. Soljacic, Photonic crystal surfaceemitting lasers enabled by an accidental Dirac point, U.S. Patent No. 8,902,946 (2 December 2014).

[71] P. O'Shea, Exceptional ring research could improve laser output by $10 \times$, https://www.electronicproducts.com/exceptionalring-research-could-improve-laser-output-by-10x/ (AspenCore, 2015), accessed 30 December 2020.

[72] C. Guo, M. Xiao, Y. Guo, L. Yuan, and S. Fan, Meron Spin Textures in Momentum Space, Phys. Rev. Lett. 124, 106103 (2020).

[73] E. M. Purcell and C. R. Pennypacker, Scattering and absorption of light by nonspherical dielectric grains, Astrophys. J. 186, 705 (1973).

[74] B. T. Draine and P. J. Flatau, Discrete-dipole approximation for scattering calculations, J. Opt. Soc. Am. A 11, 1491 (1994).

[75] A. Kwadrin and A. F. Koenderink, Diffractive stacks of metamaterial lattices with a complex unit cell: Self-consistent long-range bianisotropic interactions in experiment and theory, Phys. Rev. B 89, 045120 (2014).

[76] S. Zou, N. Janel, and G. C. Schatz, Silver nanoparticle array structures that produce remarkably narrow plasmon lineshapes, J. Chem. Phys. 120, 10871 (2004).

[77] P. Lunnemann, I. Sersic, and A. F. Koenderink, Optical properties of two-dimensional magnetoelectric point scattering lattices, Phys. Rev. B 88, 245109 (2013). 
[78] P. Lunnemann and A. F. Koenderink, Dispersion of guided modes in two-dimensional split ring lattices, Phys. Rev. B 90, 245416 (2014)

[79] S. Baur, S. Sanders, and A. Manjavacas, Hybridization of lattice resonances, ACS Nano 12, 1618 (2018).

[80] I. Sersic, C. Tuambilangana, T. Kampfrath, and A. F. Koenderink, Magnetoelectric point scattering theory for metamaterial scatterers, Phys. Rev. B 83, 245102 (2011).

[81] M. Arnold, M. Blaber, and M. Ford, Local plasmon resonances of metal-in-metal core-shells, Opt. Express 22, 3186 (2014).

[82] K. M. McPeak, S. V. Jayanti, S. J. P. Kress, S. Meyer, S. Iotti, A. Rossinelli, and D. J. Norris, Plasmonic films can easily be better: Rules and recipes, ACS Photon. 2, 326 (2015).

[83] A. Manjavacas, Anisotropic optical response of nanostructures with balanced gain and loss, ACS Photon. 3, 1301 (2016).

[84] A. Y. Song, A. R. K. Kalapala, W. Zhou, and S. Fan, Firstprinciples simulation of photonic crystal surface-emitting lasers using rigorous coupled wave analysis, Appl. Phys. Lett. 113, 041106 (2018).

[85] R. Kolkowski and A. F. Koenderink, Gain-induced scattering anomalies of diffractive metasurfaces, Nanophotonics 9, 4273 (2020).

[86] T. Savels, A. P. Mosk, and A. Lagendijk, Light scattering from three-level systems: The $T$ matrix of a point dipole with gain, Phys. Rev. A 71, 043814 (2005).

[87] W. L. Barnes, Particle plasmons: Why shape matters, Am. J. Phys. 84, 593 (2016).

[88] A. Krasnok, D. Baranov, H. Li, M.-A. Miri, F. Monticone, and A. Alù, Anomalies in light scattering, Adv. Opt. Photon. 11, 892 (2019).

[89] G. Harari, M. A. Bandres, Y. Lumer, M. C. Rechtsman, Y. D. Chong, M. Khajavikhan, D. N. Christodoulides, and M. Segev, Topological insulator laser: Theory, Science 359, eaar4003 (2018).

[90] A. Lubatsch and R. Frank, A self-consistent quantum field theory for random lasing, Appl. Sci. 9, 2477 (2019).

[91] D. Wang, A. Yang, W. Wang, Y. Hua, R. D. Schaller, G. C. Schatz, and T. W. Odom, Band-edge engineering for controlled multi-modal nanolasing in plasmonic superlattices, Nat. Nanotechnol. 12, 889 (2017).

[92] E. Plum, X.-X. Liu, V. A. Fedotov, Y. Chen, D. P. Tsai, and N. I. Zheludev, Metamaterials: Optical Activity without Chirality, Phys. Rev. Lett. 102, 113902 (2009).

[93] M. Cotrufo, C. I. Osorio, and A. F. Koenderink, Spindependent emission from arrays of planar chiral nanoantennas due to lattice and localized plasmon resonances, ACS Nano 10, 3389 (2016).
[94] J. T. Collins, C. Kuppe, D. C. Hooper, C. Sibilia, M. Centini, and V. K. Valev, Chirality and chiroptical effects in metal nanostructures: Fundamentals and current trends, Adv. Opt. Mater. 5, 1700182 (2017).

[95] T. S. H. Yoo, J. Berthelot, G. Guida, D. Demaille, E. GarciaCaurel, N. Bonod, and B. Gallas, Circularly polarized images with contrast reversal using pseudochiral metasurfaces, ACS Photon. 5, 4068 (2018).

[96] I. Sersic, M. A. van de Haar, F. B. Arango, and A. F. Koenderink, Ubiquity of Optical Activity in Planar Metamaterial Scatterers, Phys. Rev. Lett. 108, 223903 (2012).

[97] I. Fernandez-Corbaton, M. Fruhnert, and C. Rockstuhl, Objects of Maximum Electromagnetic Chirality, Phys. Rev. X 6 031013 (2016).

[98] M. Mahankali, S. Saha, N. Ghosh, and S. D. Gupta, Spectral singularity enhances transverse spin, Opt. Commun. 454, 124433 (2020).

[99] H. Li, A. Mekawy, A. Krasnok, and A. Alù, Virtual ParityTime Symmetry, Phys. Rev. Lett. 124, 193901 (2020).

[100] B. Peng, Ş. K. Özdemir, S. Rotter, H. Yilmaz, M. Liertzer, F. Monifi, C. M. Bender, F. Nori, and L. Yang, Loss-induced suppression and revival of lasing, Science 346, 328 (2014).

[101] M. Brandstetter, M. Liertzer, C. Deutsch, P. Klang, J. Schöberl, H. E. Türeci, G. Strasser, K. Unterrainer, and S. Rotter, Reversing the pump dependence of a laser at an exceptional point, Nat. Commun. 5, 4034 (2014).

[102] E. Tiguntseva, K. Koshelev, A. Furasova, P. Tonkaev, V. Mikhailovskii, E. V. Ushakova, D. G. Baranov, T. Shegai, A. A. Zakhidov, Y. Kivshar et al., Room-temperature lasing from Mie-resonant non-plasmonic nanoparticles, ACS Nano 14, 8149 (2020).

[103] M. Benzaouia, A. Cerjan, and S. G. Johnson, Is single-mode lasing possible in an infinite periodic system? Appl. Phys. Lett. 117, 051102 (2020).

[104] A. Cerjan and S. Fan, Eigenvalue dynamics in the presence of nonuniform gain and loss, Phys. Rev. A 94, 033857 (2016).

[105] H. Schomerus, Quantum Noise and Self-Sustained Radiation of $\mathcal{P} \mathcal{T}$-Symmetric Systems, Phys. Rev. Lett. 104, 233601 (2010).

[106] M. Parto, W. Hayenga, A. Marandi, D. N. Christodoulides, and M. Khajavikhan, Realizing spin Hamiltonians in nanoscale active photonic lattices, Nat. Mater. 19, 725 (2020).

[107] P. P. Ewald, Die Berechnung optischer und elektrostatischer Gitterpotentiale, Ann. Phys. (Berlin) 369, 253 (1921).

[108] K. E. Jordan, G. R. Richter, and P. Sheng, An efficient numerical evaluation of the Green's function for the Helmholtz operator on periodic structures, J. Comput. Phys. 63, 222 (1986). 